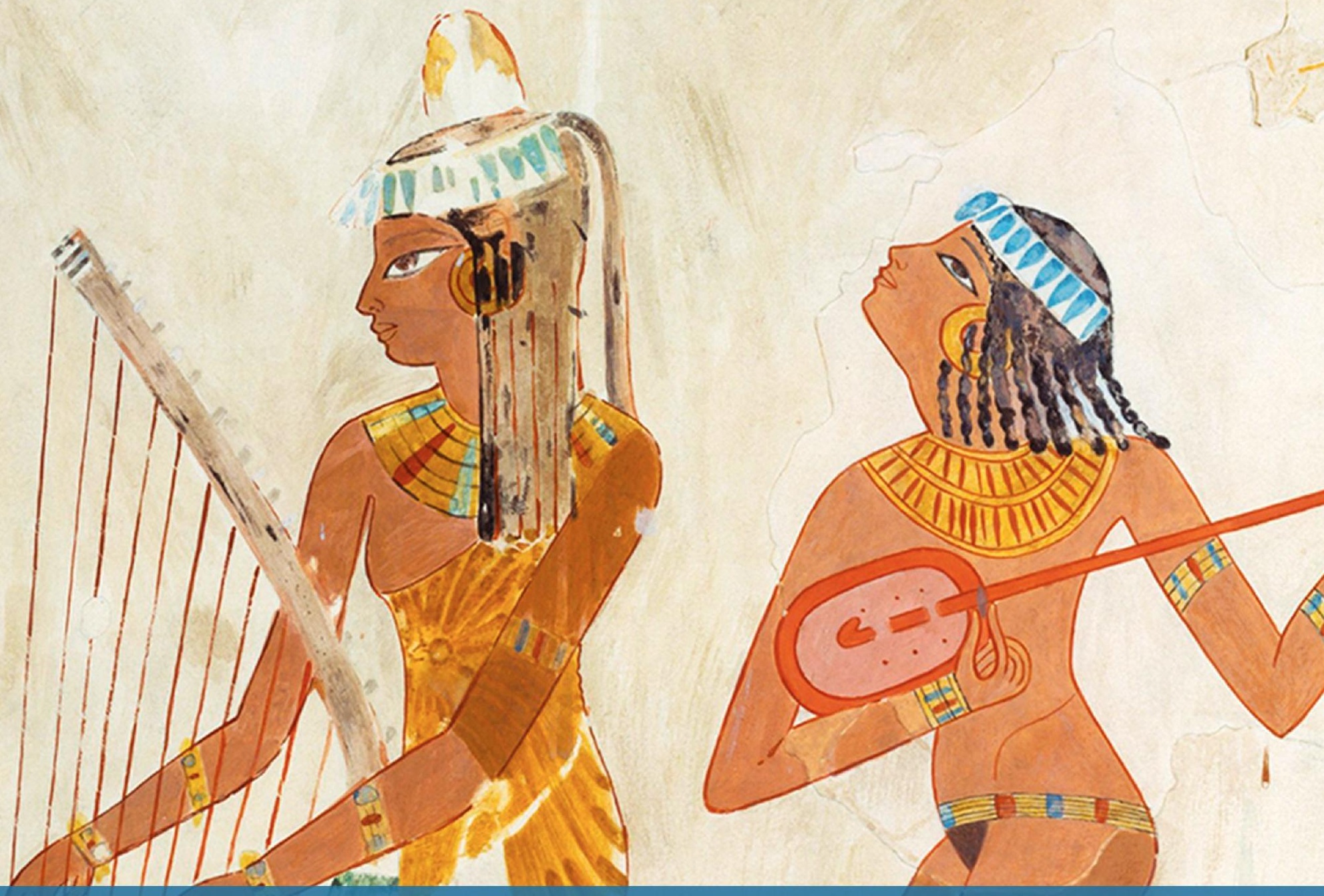

Revista digital de ciencia y Didáctica de la Historia

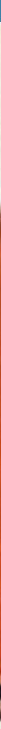




\section{Panta Rei \\ Revista Digital de Ciencia \\ y Didáctica de la Historia}

\section{9}

Revista anual

Fecha de inicio: 1995

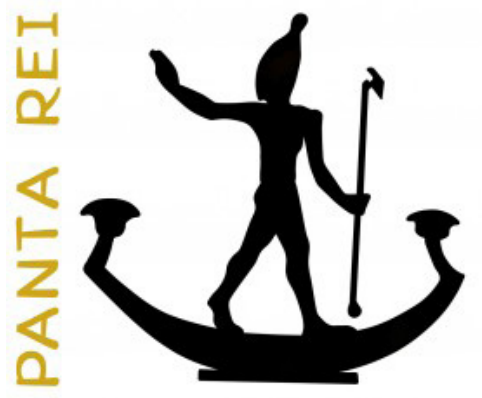

Revista Panta Rei. pantarei@um.es

Edita:

Centro de Estudios del Próximo Oriente y la

Antigüedad Tardía - CEPOAT

Edificio Universitario Saavedra Fajardo.

Universidad de Murcia

C/ Actor Isidoro Máiquez, 9

30007 - MURCIA - ESPAÑA

Teléfono: (+34) 868883890

cepoat@um.es

Web: www.um.es/cepoat/pantarei

Edición 2019

ISSNe: 2386-8864

Responsable de la presente edición: Consejo Editorial Panta Rei.

UNIVERSIDAD DE MURCIA centro de estudios del próximo oriente y la antigüedad tardía

En Portada: Fragmento de pintura de la tumba de Djeserkareseneb (TT38), Tebas. (fotografía del Metropolitan Museum).

Responsables de los textos: Sus autores.

ISSN: 1136-2464

Depósito legal: MU-966-1995 


\section{CONSEJO DE REDACCIÓN}

\section{Coordinador editorial}

Egea Vivancos, Alejandro

[Didáctica de las Ciencias Sociales, UMU]

\section{Editores}

Jiménez Vialás, Helena

[UMU]

López Muñoz, Dámaris

[UJA]

Meseguer Gil, Antonio José

[CEPOAT, UNED]

Sáez Giménez, David Omar

[CEPOAT, UMU]

Sánchez Mondéjar, Celso Miguel

[Patrimonio Inteligente]

\section{Secretaria}

Arias Ferrer, Laura

[Didáctica de las Ciencias Sociales, UMU]

\section{Responsable informático}

Martínez García, José Javier

[CEPOAT, UMU]

\section{Traducción y corrección lingüística}

Martínez Martínez, Cristina

[Sociedad Española de Lenguas Modernas]

Albaladejo Albaladejo, Sara

[ISEN, UMU]

\section{CONSEJO ASESOR}

Adroher Auroux, Andrés María [Arqueología, Universidad de Granada]

Albero Muñoz, M. ${ }^{a}$ del Mar [Historia del Arte, Universidad de Murcia]

Alia Miranda, Francisco [Historia Contemporánea, UCLM]

Arciniega García, Luis [Historia del Arte, Universidad de Valencia]

Barrio Barrio, Juan Antonio [Historia Medieval, Universidad de Alicante]

Castellano i Solé, Núria [Egiptología, Schola Didàctica Activa S.L.]
Chapman, Arthur [History Education, University College of London, Reino Unido]

Cid López, Rosa María [Historia Antigua, Universidad de Oviedo]

Cobacho López, Ángel [Derecho, Universidad de Murcia]

Cuenca López, José María [Didáctica de las Ciencias Sociales, Universidad de Huelva]

Egea Bruno, Pedro M. ${ }^{a}$ [Historia Contemporánea, Universidad de Murcia]

Feijoo Martínez, Santiago [Arqueología, Consorcio Ciudad Monumental de Mérida]

García Atienzar, Gabriel [Prehistoria, Universidad de Alicante]

Ginestí Rosell, Anna [Filología Clásica, Katholische Universität Eichstätt-Ingolstadt]

González Monfort, Neus [Didáctica de las Ciencias Sociales, Universidad Autónoma de Barcelona]

González Soutelo, Silvia [Arqueología, Universidad de Vigo]

Haber Uriarte, María [Prehistoria, Universidad de Murcia]

Hernández de la Fuente, David [Historia Antigua, Universidad Complutense]

Hutson, Scott R. [Anthropology, University of Kentucky, EE UU]

Igual Luis, David [Historia Medieval, UCLM]

Irigoyen López, Antonio [Historia Moderna, Universidad de Murcia]

Jover Maestre, Francisco Javier [Prehistoria, Universidad de Alicante]

Mahony, Simon [Digital Humanities, University College of London, Reino Unido]

Marsilla de Pascual, Francisco Reyes [Técnicas historiográficas, Universidad de Murcia]

Martínez-Burgos García, Palma [Historia del Arte, UCLM]

Mathis, Christian [Didaktik der Geschichte, PH Zürich]

Miralles Maldonado, José Carlos [Filología Clásica, Universidad de Murcia]

Molina Gómez, José Antonio [Historia Antigua, Universidad de Murcia]

Mónica Ghirardi [Historia Moderna, Universidad Nacional de Córdoba, Argentina]

Navarro Espinach, Germán [Historia Medieval, Universidad de Zaragoza]

Noguera Celdrán, José Miguel [Arqueología, Universidad de Murcia]

Ortiz Heras, Manuel [Historia Contemporánea, UCLM]

Panzram, Sabine [Historia Antigua, Universität Hamburg]

Pérez Molina, Miguel Emilio [Filología Clásica, Universidad de Murcia]

Prados Martínez, Fernando [Arqueología, Universidad de Alicante]

Sánchez Ibáñez, Raquel [Didáctica de las Ciencias Sociales, Universidad de Murcia]

Sancho Gómez, Miguel Pablo [Educación, UCAM]

Victoria Moreno, Diego [Historia Contemporánea, UNED]

Vilar García, María José [Historia Contemporánea, Universidad de Murcia]

Vivas Sainz, Inmaculada [Historia del Arte, UNED]

Zamora López, José Ángel [Próximo Oriente Antiguo, CCHS-CSIC] 



\section{Índice}

\section{Artículos}

La imagen de la Prehistoria en el cine y los géneros del cine prehistórico. Un mundo de hombres mono, bikinis y dinosaurios.

Alberto Lombo Montañés.

Trabajo y roles de género durante la Prehistoria. Un estudio sobre su percepción en el alumnado de Historia.

María Pastor Quiles y Daniel Mateo Corredor.

Memoria cultural en el Egipto Faraónico. Algunas reflexiones sobre su origen, función y pervivencia histórica.

Antonio Pérez Largacha.

La representación de la danza dentro de las escenas de banquete de las tumbas tebanas privadas de la XVIII dinastía egipcia.

Miriam Bueno Guardia.

La "Sala del Fresco" de Micenas. Revisión de las interpretaciones del programa iconográfico y nueva lectura en relación a los espacios.

Pelayo Huerta Segovia.

Las advertencias de Quilón y Solón sobre la tiranía de Pisístrato.

Unai Iriarte.

La Virgen de la Leche. Arquetipo de mujer y madre en la pintura del Renacimiento español.

Patricia Castiñeyra Fernández

Innovación didáctica en Historia: un estado de la cuestión en torno a cuatro ejes temáticos.

Diego Luna Delgado.

\section{Reseñas}

Foster, B. R. (2016), The Age of Agade. Inventing empire in ancient Mesopotamia, Londres y Nueva York: Routledge, 438 págs.

Juan Álvarez García.

Alviz Fernández, M. y Hernández de la Fuente, D. (Eds.) (2017). De ópos a limes: el concepto de frontera en el mundo antiguo y su recepción. Madrid: Escolar y Mayo editores. 256 págs.

Ethan Yepes de la Hoz.

Clares Clares, M ${ }^{\mathrm{a}}$ E. (2017). Música y noches de moda. Sociedades, cafés y salones domésticos de Murcia en el siglo XIX. Murcia: Universidad de Murcia, Servicio de Publicaciones. 474 págs.

Elena Micó Terol 195

Goñi Zabalegui, A. (2018). Género y sociedad en el Egipto romano. Una Mirada desde las cartas de mujeres. Oviedo: Ediciones de la Universidad de Oviedo. 360 págs.

Alejandra Izquierdo Perales. 199

Normas de publicación/Publishing rules 



\title{
Innovación didáctica en Historia: un estado de la cuestión en torno a cuatro ejes temáticos
}

\author{
Innovative Teaching Approaches in History: A Study Based on Four Central Themes \\ Luna Delgado, Diego 1 \\ Universidad de Sevilla
}

Recibido: 04/01/2019

Aceptado: 04/03/2019

Para citar este artículo: Luna Delgado, D. (2019). Innovación didáctica en Historia: un estado de la cuestión en torno a cuatro ejes temáticos. Panta Rei. Revista Digital de Ciencia y Didáctica de la Historia, 161-181.

ISSNe: $2386-8864$

DOI: $10.6018 /$ pantarei/2019/08

\begin{abstract}
Resumen
El objetivo de este trabajo es ofrecer una panorámica a través de las diferentes perspectivas teóricas desde las que se ha venido abordando la cuestión de la innovación didáctica en la materia de Historia durante los últimos quince años. Para ello se elabora una síntesis descriptiva dividida en cuatro ejes temáticos (herramientas digitales, nuevas metodologías, transformación de la cultura docente y evaluación de la innovación), destacando una serie de conceptos y experiencias empíricas en cada uno de ellos. Entre otras cosas, tal procedimiento revela que la motivación del alumnado es el centro de interés tanto de este campo de investigación como del modelo educativo que hoy se está intentando implementar. Sin embargo, frente al exceso de optimismo y actitudes acríticas detectado respecto a este último, se defiende la necesidad de aumentar la rigurosidad a la hora de comparar discursos innovadores y prácticas educativas.
\end{abstract}

\section{Palabras clave}

Enseñanza de la Historia, innovación educativa, innovación docente, revisión de la literatura, motivación del alumno.

\begin{abstract}
The aim of this paper is to offer an insight into innovative teaching approaches in History, based on the different theoretical perspectives that have been tackled over the last 15 years. In order to achieve this purpose, the paper includes a descriptive summary divided into four central themes (digital tools, new methodologies, transformation of teaching culture and evaluation of innovation), with particular emphasis on a series of concepts and empirical experiences. Among other things, the study reveals that students' motivation is of the upmost importance not only in this study, but also in the education model which is currently being sought implement. When faced, however, with overoptimism and a lack of criticism of said model, there arises the need for a more rigorous discussion to compare innovative discourses and educational practices.
\end{abstract}

1 Para contactar con el autor: Diego Luna Delgado. Universidad de Sevilla. dielundel@gmail.com. 


\section{Keywords}

History instruction, educational innovation, instructional innovation, literature reviews, student motivation.

\section{Introducción}

El encuentro entre tradición e innovación en la escuela del siglo XXI constituye una problemática del todo relevante en el ámbito de la enseñanza y el aprendizaje de la Historia, terreno donde la óptica adoptada resulta especialmente determinante a la hora de captar el interés del alumnado y, por tanto, de generar procesos educativos valiosos y satisfactorios para todas las partes implicadas. En líneas generales, la cultura escolar predominante en nuestros días podría entenderse como una concreción práctica de un discurso global en el que la innovación se ha convertido en el objeto de deseo por excelencia. Cada vez son más las personas que identifican el modelo educativo innovador (defensor de la participación, del uso de herramientas digitales, de metodologías abiertas a la creatividad y al pensamiento crítico, etc.) con una cuestión de prestigio, como ocurre en realidad con muchos otros fenómenos basados en el principio de innovar por innovar.

En estas tesituras se enmarca el trabajo al que se adscribe la revisión bibliográfica que se presenta, centrado precisamente en el choque entre los modelos didácticos tradicional e innovador en el contexto de la clase de Historia. En otras palabras, de lo que se trata es de identificar y analizar las consecuencias prácticas de la confluencia en el aula de dos paradigmas teóricos y metodológicos, es decir, de dos maneras bien distintas de plantear los procesos educativos. De ahí que las dos preguntas de investigación en él planteadas sean: por un lado, ¿cómo es vivido el choque entre tradición e innovación en la clase de Historia por parte del profesor y su alumnado?; y, por otro, ¿qué resultados se extraerían del diseño y la experimentación de un "ámbito de investigación escolar" sobre educación mediática que intentase superar algunas de las carencias y dificultades previamente detectadas ? ${ }^{2}$. Para ello se ha optado por una metodología de investigación-acción con estudio de caso, apoyada en el método etnográfico mediante las técnicas de la entrevista semiestructurada y la observación participante, así como en el análisis crítico del discurso aplicado a todos los elementos que condicionan el desarrollo de los procesos de enseñanza-aprendizaje en el contexto mencionado (currículo, filosofía pedagógica del centro, orientaciones metodológicas del departamento, roles del profesor y del estudiante, etc.). A través de este proceso inductivo se pretende formular una teoría compleja que no solo describa las convergencias y divergencias entre una cultura escolar que se resiste a desaparecer y otra que se intenta implementar, sino que también aporte a otros docentes e investigadores un conocimiento útil para la superación de las posibles dificultades (académicas, organizativas, disciplinarias, sociales, etc.) emanadas de tal encuentro. De lo que se trata es por tanto de deconstruir el dispositivo educativo tejido entre los participantes y el contexto discursivo del que estos extraen y al que estos aportan nuevos significados, por lo que desde un primer momento resultó fundamental conocer la manera en que dicho dispositivo, el caso de estudio en sí mismo, había sido ya abordado por otros investigadores.

Ante todo, es preciso constatar que el paradigma educativo innovador es uno de los temas estrella de la investigación en educación y prueba de ello es la cantidad y variedad de publicaciones que ha inspirado en los últimos tiempos. Así lo demuestra una búsqueda rápida en ProQuest, utilizando datos de ERIC, en cuyos criterios se puede observar una gráfica que refleja un aumento exponencial de publicaciones dedicadas al tema a partir del año 2000 , y específicamente a partir de 2010 , respecto a la situación anterior (destacando siempre la gran productividad de los años setenta del pasado siglo) (Figura 1). Todas ellas conforman un vasto corpus que, sin embargo, se reduce

2 "Desde la perspectiva de IRES, los 'ámbitos de investigación escolar' son conjuntos de problemas socioambientales que actúan como organizadores del currículo, son relevantes para la comprensión de la realidad e integran, definen y concretan el conocimiento escolar deseable" (Pineda-Alfonso y García Pérez, 2011, p. 83). 
drásticamente si, acorde con los parámetros teóricos y metodológicos establecidos en la investigación global descrita hace un momento, requerimos a los trabajos las siguientes características: 1) que comparen las prácticas educativas tradicionales e innovadoras, reflexionando sobre las diferencias entre unas y otras; 2) que sean de corte cualitativo y adopten la investigación-acción con estudio de caso como metodología; y 3) que tomen como caso la asignatura de Historia en la Enseñanza Secundaria Obligatoria desde la perspectiva de su didáctica. Frente a estos parámetros de búsqueda, lo que hallamos son principalmente abundantes investigaciones enmarcadas en los ámbitos de la educación Infantil, Primaria y Superior, centradas en las clases de Matemáticas o de Lengua y Literatura, e incluso en el potencial deweyano de la clase de Historia como laboratorio democrático.

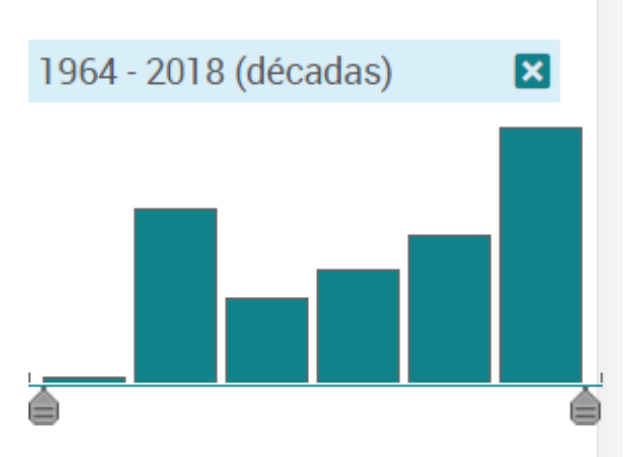

Figura 1: evolución cuantitativa de las publicaciones internacionales sobre innovación educativa desde 1964 hasta 2018, según la base de datos ERIC. Fuente: ProQuest.

\section{Objetivos y metodología}

El objetivo de este trabajo no es otro que el de describir las principales vías de investigación a través de las cuales diferentes especialistas han venido abordando la innovación didáctica en la materia de Historia durante los últimos quince años. Con ello se pretende definir unos primeros asideros teóricos que, gracias a su conexión directa con las preguntas de investigación planteadas en el proyecto marco, orienten el desarrollo global del mismo, al tiempo que también puedan ayudar a otros docentes e investigadores a contextualizar sus propias investigaciones en este terreno. En este sentido se ha hecho especial hincapié en la identificación de dimensiones relevantes para el análisis de nuestro caso de estudio, la clase de Historia, surgidas gracias al proceder emergente adoptado a la hora de analizar la bibliografía seleccionada, y en torno a las cuales se estructura el núcleo de este informe. Asimismo, mediante su elaboración se ha intentado: hallar las relaciones entre ciertos conceptos y determinadas aplicaciones prácticas, evitar posibles aproximaciones infructuosas, conocer cuáles eran los aspectos más controvertidos del tema, proporcionar resultados con los que comparar nuestros propios hallazgos, comprender qué tipo de aportación puede o debe constituir nuestra investigación al área de la didáctica de la Historia y, finalmente, formular nuevas preguntas que puedan dar lugar a futuras investigaciones.

En lo que respecta a la metodología empleada, cabría advertir de antemano que, más allá de una serie de tesis doctorales que en los últimos años han abordado aspectos más o menos parciales del tema (Ansó, 2017; De Miguel, 2014; Duarte, 2015; Fernández, 2016; Haro, 2015; Riera, 2010), la ausencia de investigaciones completamente replicables en el contexto elegido dificultaría sobremanera la elaboración de un marco teórico al uso. Ante el carácter holístico que se pretendía adoptar, la solución ha pasado por rastrear separadamente aquellos elementos teóricos más próximos al caso de estudio, para lo cual algunas de las fuentes secundarias y terciarias más utilizadas han sido las siguientes: revistas de alto impacto (recogidas en WOS o SCOPUS); trabajos colectivos surgidos a partir de proyectos de investigación o encargos institucionales (López Torres, García Ruíz y Sánchez Agustí, 2018; Maquilón, García-Sanz y Belmonte, 2015; Marcelo, 2011; 
Medina, 2015); o bases de datos como ERIC, ProQuest o Dialnet, ajustando sus respectivos filtros (en los casos en que esto era posible) hacia la búsqueda de trabajos "evaluados por expertos", realizados en el marco temporal 2005-2018 y relativos a niveles de educación media.

A continuación se recoge la panorámica resultante del análisis de una serie de conceptos y experiencias empíricas que permiten arrojar luz a los objetivos expresados. Estos textos han sido clasificados en las cuatro líneas temáticas emergidas durante el mismo proceso de revisión bibliográfica, estrecha e inevitablemente conectadas, pero garantes por separado de lo que podríamos entender como cuatro ejes o dimensiones específicas de la cuestión de la didáctica de la Historia en la actualidad: 1) herramientas digitales, incluyendo conceptos como los de EVA (Entorno Virtual de Aprendizaje) o TAC (Tecnologías del Aprendizaje y el Conocimiento); 2) nuevas metodologías, tales como el aula invertida [flipped classroom], el PBL [Project-based Learning], el PBL [Problembased Learning], el aprendizaje cooperativo, las rutinas de pensamiento, la gamificación, etc.; 3) cultura docente, desde la formación del profesorado frente a los procesos innovadores o desde la perspectiva TPACK [Technological Pedagogical Content Knowledge]; y, por último, 4) evaluación de la innovación, en base a la comparación de los contextos previos y posteriores a la implementación de alguna estrategia de este tipo. Cada uno de estos bloques temáticos funciona así como una red de significados y prácticas en constante fricción con las otras redes, confluyentes igualmente en el ámbito que comporta la clase de Historia. Grandes conjuntos de elementos heterogéneos que, bajo la influencia del mismo discurso innovador, aglutinan a su vez otros tantos aspectos temáticos que, aunque protagonistas quizá en otras circunstancias (los contenidos curriculares, las fuentes históricas, los valores cívicos y ciudadanos, la sensibilización patrimonial, etc.) aparecen hoy diseminados a través de ellos.

\section{Resultados}

\subsection{Herramientas digitales}

En un primer bloque debemos incluir todos aquellos trabajos que analizan el potencial pedagógico y didáctico de un conjunto de elementos que, aunque referidos en el mismo discurso de la innovación que ayudan a mantener, cumplen una función que podríamos denominar sustentante -soportes y códigos- dentro de los procesos particulares de enseñanza y aprendizaje de la Historia. En esta ocasión se trata de una variada gama de lenguajes, herramientas, entornos y medios, surgidos a partir de la consolidación de Internet como entidad comunicativa matriz a finales del siglo XX. La característica común y más relevante desde el punto de vista técnico es que todos ellos poseen y operan en una dimensión digital, permitiendo a cualquier individuo con un mínimo de conocimiento informático crear, manipular y compartir con facilidad nuevos recursos. La interactividad, la reutilización de otros materiales y las posibilidades de andamiaje que ofrece tal carácter digital son algunos de los factores por los que estos han sido especialmente recomendados para el diseño de los denominados "objetos de aprendizaje" (Elliott y Sweeney, 2008; Haughey y Muirhead, 2005). Subrayando su revolucionaria novedad, Bolick (2006) expresaba: "Digital archives democratize the doing of history and provide social studies educators with an opportunity to engage students in constructivist-based learning in a manner that was practically impossible before the advent of the World Wide Web" (p. 133).

Dentro de este grupo de trabajos, no es casualidad que uno de los aspectos más investigados haya sido el del medio fílmico y todos los lenguajes a él asociados, entendidos estos como recursos válidos tanto para el profesor como para el alumnado por su consonancia con una cultura, como la actual, donde el audiovisual y sus códigos constituyen el lenguaje comunicativo predominante (Kearney y Schuck, 2006; Schul, 2012, 2014). En términos metodológicos, el vídeo resulta en efecto muy útil para fomentar un tipo de proceso educativo activo, experiencial y reflexivo (Stoddard, 2012; Stoddard y Marcus, 2010; Woelders, 2007), un "auténtico trabajo intelectual" (Swan, Hofer y Swan, 2011; Swan y Hofer, 2013), pero también, gracias precisamente a todo ello contribuye al desarrollo 
de proyectos cooperativos. Este hecho se ha visto favorecido por la gran difusión de dispositivos como los smartphones o las tabletas electrónicas (Cheung y Hew, 2009) y, en general, al potencial interactivo de Internet, la Web 2.0 y su característica lógica hipertextual, que permiten intercambiar materiales con facilidad y acceder a aplicaciones de edición altamente intuitivas y, por tanto, de fácil manejo para los estudiantes (Verezub, Grossi, Howard y Watkins, 2008). Como concluía Bolick (2006):

The fact that there was not one body of knowledge that they were expected to learn and one way to learn it was atypical for the teachers both as graduate students and teachers. The hypertext inquiry activity provided a more open, constructivist, and democratic learning environment than one that is teacher-and knowledge-centered ( $p$. 132).

En relación a la segunda generación de recursos de Internet, son muchos los autores que han subrayado el potencial de este formato de cara al funcionamiento de blogs y fotoblogs (Barrow, Anderson y Horner, 2017), redes sociales (que algunos denominan incluso Web 3.0), wikis (Heafner y Friedman, 2008), podcasts (Lipscomb, Guenther y McLeod, 2007), screencasts (Snyder, Paska y Besozzi, 2014), vodcasts (Gkatzidou y Pearson, 2009) y, en general, cualquier plataforma o recurso web planteado desde un punto de vista eminentemente creativo y colaborativo, enfocado hacia la compartición de información en cualquier formato de archivo (Kingsley y Boone, 2008; Wilson, Wright, Inman y Matherson, 2011). En el marco de las infinitas posibilidades que ofrece la Web 2.0 para la renovación de la didáctica de la Historia (Diacopoulos, 2015; Kingsley y Brinkerhoff, 2011), algunos investigadores se han centrado en el uso por parte de muchos docentes de los denominados webquests, una herramienta tanto de aprendizaje, por medio del uso de materiales de la Red, como de evaluación, en la medida en que constituyen espacios sencillos de supervisar; pero sobre todo un instrumento atractivo para la adquisición de autonomía del alumnado, con el aliciente de que este puede recibir un feedback instantáneo (Bates, 2008; Butchart et al., 2009; Crocco y Cramer, 2005).

Por otro lado, cada vez son más los autores que, desde diferentes ámbitos de investigación, se dedican a destacar y reivindicar el potencial pedagógico de un fenómeno que hasta hace poco tiempo había estado relegado de la forma más peyorativa a la industria del ocio, entendido como ámbito de mero entretenimiento: los videojuegos. Algunos de los aspectos más destacados de ellos son su potencial colaborativo (Lim y Wang, 2005) y sus cualidades estéticas e interactivas, que hacen de estos nuevos recursos unas herramientas sin parangón para despertar hoy el interés de los estudiantes (Cuenca y Martín, 2010; Lee y Probert, 2010; Pagnotti y Russell, 2012). Muy ligado al uso educativo de este tipo de herramientas multimedia, aunque constituyendo quizá algo incluso más novedoso, se encuentran los denominados "entornos virtuales de aprendizaje" (EVA, $V L E$ en inglés), es decir, ambientes cibernéticos que favorecen la interacción y el desarrollo tanto cognitivo como tecnológico de los diferentes actores implicados en un mismo proceso educativo (Rauch, Cohodas y Wang, 2009; Zieger y Farber, 2012). Inmersiva o no, algunos investigadores han confirmado que la realidad virtual será en los próximos años el medio decisivo para el diseño de los EVA (Chen, 2006; Ellison y Matthews, 2010). En todo caso, redes sociales como Facebook o Twitter han sido las últimas herramientas en ser reivindicadas por docentes, pedagogos e investigadores, ya no solo por el carácter participativo e instantáneo que las define (Bull y Adams, 2012; Cheong y Cheung, 2008), sino también por su capacidad para mejorar tanto las relaciones entre alumnado y profesores (King-yin y Trinidad, 2005) como el pensamiento crítico mediante la participación en debates grupales. A este respecto, Parry (2008) reconocía más de una decena de razones por las que el hecho de haber introducido Twitter en su clase había merecido la pena.

De un modo u otro, la confluencia de todos estos variados y novedosos elementos tecnológicos (soportes, códigos, lenguajes, dispositivos, herramientas web, etc.) permite constatar la aparición de lo que podríamos definir como un revolucionario ecosistema para el aprendizaje y la enseñanza de la Historia en el siglo XXI. La idea de fondo es que cada uno de estos avances forma parte de 
un nuevo paradigma mediológico cuyas primeras repercusiones en el ámbito escolar ya han sido identificadas por los investigadores educativos: la activación efectiva del discente gracias al aumento de las posibilidades creativas; el apoyo a la dimensión cooperativa del aprendizaje mediante el uso de formatos basados específicamente en una filosofía de corte colaborativo; o el fuerte componente motivacional que todo lo anterior trae consigo. Sin embargo, aún quedaría un aspecto determinante por subrayar y que requeriría de una reubicación del tipo de mejoras detectado en un marco de análisis mucho más amplio y complejo. Dicho en pocas palabras, lo verdaderamente interesante de las innovaciones tecnológicas en relación a la práctica de la enseñanza y el aprendizaje de la historia sería la transformación de tipo epistemológico que favorecen: dejar de abordar la historia desde una óptica eminentemente transmisiva y pasiva trae consigo una concepción de ella como un terreno abierto al replanteamiento crítico y a la vez autocrítico, como un espacio propicio para la construcción de relatos genealógicos aún no necesariamente transitados; en definitiva: una democratización de la historia.

\subsection{Nuevas metodologías}

En un segundo bloque de investigaciones, lo primero que llama la atención es un interés didáctico por vincular o aplicar metodologías innovadoras al tratamiento de determinadas problemáticas e inquietudes sociales en la clase de Historia. Así, por ejemplo, podemos encontrar trabajos que tratan el diseño de enfoques metodológicos dirigidos hacia la integración de determinados colectivos étnicos (Olukayode y Salako, 2014) o, en general, hacia la inclusión social (Connor y Lagares, 2007). Junto a ellos hallamos temas de gran actualidad, tales como la reivindicación de un tipo de metodología activa y personalizada, centrada en las capacidades y los intereses del alumnado (Aponte-Martínez y Pellegrino, 2017) o en las virtudes del trabajo con la cultura material para comprometer a este en su propio proceso de aprendizaje (Brewer y Fritzer, 2011; Egea, Arias y Santacana, 2018). Una de las cuestiones que más ha contribuido al desarrollo de reflexiones sobre metodología diseñada para alcanzar una mayor autonomía del alumno, en este caso mediante la reivindicación de la importancia del descubrimiento, ha sido sin duda la de la denominada "clase invertida" [flipped classroom] (Mazur, Brown y Jacobsen, 2015), una reformulación espaciotemporal de la clase por la cual se fomenta el trabajo individual del discente fuera de la escuela. Relacionados igualmente con la dimensión proactiva de las estrategias innovadoras, hay que dedicar una mención especial a aquellos trabajos que analizan el impacto de las mismas en el desarrollo del alumnado que presenta dificultades para el aprendizaje. En esta línea, destacan textos como el de Haydon, Maheady y Hunter (2010), que confirman la mejora de los resultados de este tipo de alumnado en concursos y tareas diarias mediante la implementación de la estrategia Numbered Heads Together en la clase de Historia, dentro de una reivindicación global de las metodologías que implican o determinan la realización de prácticas grupales. Del mismo modo, habría que mencionar interesantes trabajos centrados en la lectura colaborativa (Boardman et al., 2016), en la investigación online (Castek, Coiro, Guzniczak y Bradshaw, 2012) o en el aprendizaje basado en proyectos, mediante herramientas como los blogs, las wikis, las redes sociales o el video cooperativo (O'Brien y Wood, 2011; Tinnerman, Johnson y Grimes, 2010).

En todo caso, las investigaciones sobre aspectos metodológicos pueden ordenarse siguiendo un criterio de sofisticación -que no de complejidad- del objeto de análisis. Aspectos que, en ocasiones, han sido abordados lógicamente sobre el trasfondo tecnológico actual o, al revés, sobre la importancia de la tecnología desde el punto de vista metodológico (Keeler, 2008). De esta forma, podemos observar estudios y reflexiones que van desde el abordaje de elementos tan sutiles como el propio concepto de idea (Foels, 2009), de pregunta (Kracl, 2012) o de técnicas de organización conceptual (Gallavan y Kottler, 2007), hasta la observación de experiencias reales en la clase de Historia donde se han empleado alguna técnica o procedimiento novedoso para implicar o aumentar el interés del alumnado. Destacan en este sentido las denominadas "estaciones de aprendizaje" (Ediger, 2011), donde el estudiante elige las actividades que quiere realizar, o la propia investigación para mejorar la comprensión de los textos (Swanson y Wanzek, 2014). Un caso 
bastante llamativo al respecto de todo ello es el de las simulaciones (De Frutos, 2015), fórmulas de aprendizaje experiencial donde las haya y sin duda relacionadas con las técnicas de recreación histórica propias de la living History o el Historical reenactment, tanto de episodios concretos (WrightMaley, 2014) como de personajes (Daniels, 2010; Rowland, 2010), mediante la dramatización o role-playing (Worthington, 2018) y herramientas tan útiles como el croma (Sheffield y Swan, 2012) o los videojuegos (Devlin-Scherer y Sardone, 2010). Finalmente, la investigación educativa no ha permanecido ni mucho menos ajena al protagonismo crucial que ha adquirido en la última década la cuestión del aprendizaje basado en proyectos y sus múltiples variantes como posibilidades metodológicas (Garran, 2008; Hernández-Ramos y De la Paz, 2009). Relacionado con ello por motivos obvios, las dos metodologías que han captado más la atención de los investigadores, dentro del corpus bibliográfico dedicado específicamente a la metodología en la enseñanza de la historia, han sido sin duda alguna el aprendizaje basado en problemas (Abdu-Raheem, 2012; Yukhymenko, Brown, Lawless, Brodowinska y Mullin, 2014) y, sobre todo, el aprendizaje cooperativo (Adeyemi, 2008; Salako, Eze y Adu, 2013; Yusmanto, Soetkipto y Djatmika, 2017).

Como puede observarse, las metodologías innovadoras constituyen una serie de estrategias que, apoyadas en las nuevas herramientas y lenguajes, tienen como objetivo reconfigurar, en nuestro caso, los procesos de aprendizaje de la Historia. De entre los elementos que las constituyen destacan aspectos como los de la pregunta, la autonomía del discente o el trabajo cooperativo, factores que determinan su naturaleza eminentemente activa y experiencial. Se pregunta sobre hechos del pasado pero a partir de problemáticas del presente; se aprende historia pero al ritmo que marca el propio alumno. La aplicación de técnicas y métodos perfectamente complementarios entre sí, tales como la clase invertida o la lectura compartida, contribuyen en este sentido al desarrollo de una enseñanza y un aprendizaje multifocales de la Historia, lo que contribuye a desarrollar ese cambio radical de perspectiva frente a la misma, indicado anteriormente a propósito de las nuevas herramientas empleadas. El conocimiento que se busca conseguir no depende ya de la memorización de un discurso cerrado, sino de la capacidad de reconstruir el pasado desde una realidad, como la actual, que ofrece una serie de medios idóneos para ello. Este giro metodológico hacia la conversión del alumno en "un aprendiz de historiador" (Miralles y Rodríguez, 2015, p. 71) se encuentra en autores como Sam Winebourg, si bien la enseñanza de la Historia de una manera reconstructora y activa en general fue ya introducida en los años setenta del pasado siglo de la mano de Peter J. Lee y la escuela anglosajona. Esta perspectiva didáctica es ahora favorecida por el nuevo ecosistema tecnológico (Bull, Hammond y Fester, 2008), abriendo todo un campo de oportunidades para aquellos estudiantes que presenten dificultades cognitivas, aunque generando otros tantos nuevos retos en términos de evaluación del aprendizaje.

\subsection{Cultura docente}

En un tercer grupo de trabajos nos encontramos todas aquellas investigaciones que abordan la cuestión de la innovación educativa en la materia de Historia desde la dimensión de la formación y la cultura docentes, es decir, cómo se enfrentan los profesores a las propuestas innovadoras y en qué medida estas cambian su práctica profesional. Debido a la madurez que se les presupone respecto a los discentes, los docentes son por definición aquellos agentes cuyas opiniones sobre los procesos educativos poseen un mayor interés para los investigadores, siendo a menudo privilegiadas por encima de las del alumnado (Boon, Fore y Spencer, 2007; Cengelci, 2013; Zhao, 2007). Artículos como el de Binkley, Keiser y Strahan (2011) resultan especialmente atractivos por emplear una metodología enfocada específicamente hacia la descripción y el análisis de los procesos innovadores desde este enfoque, pero también lógicamente por los propios resultados obtenidos. En este caso, las tres experiencias estudiadas constituyen tres formas distintas de integrar la enseñanza de la Historia con el crecimiento profesional del docente. En esta línea, Martell y Sequenzia (2016) se centraban en dos experiencias guiadas por el lema "trabajar la dialéctica", con el fin de conocer el impacto del aprendizaje previo de la investigación docente como herramienta para la mejora de 
la enseñanza de los valores cívicos y democráticos. Como no podía ser de otra manera, no faltan los textos que subrayan el interés de determinados docentes por implementar esta misma actitud investigadora en su alumnado, mediante proyectos de elaboración y difusión de trabajos que parten de un consenso en torno a las propias preguntas de investigación (Virtue, Buchanan y Vogler, 2012).

Todo ello parece tener que ver con uno de los nuevos requisitos de la práctica docente: el de seducir a los discentes. Sin un reconocimiento directo, conseguir y mantener la motivación o la atención se ha erigido en uno de los objetivos curriculares para los docentes actuales (Anderman, Andrzejewski y Allen, 2011). Trabajos como el de Grant y Gradwell (2009) son verdaderamente sugerentes en este sentido por explorar, en su caso, las estrategias empleadas por cuatro profesores para desarrollar una "enseñanza ambiciosa" mediante el uso de "grandes ideas" para trabajar la Historia. Un planteamiento desafiante que, según los autores, favorece el aprendizaje complejo, significativo, variado, flexible y centrado en los intereses del alumno. El juego como recurso didáctico ha sido a este respecto uno de los asuntos que más ha atraído a autores como Chee, Mehrotra y Ong, quienes han dedicado varios artículos (Chee, Mehrotra y Liu, 2013; Chee, Mehrotra y Ong, 2015a, $2015 \mathrm{~b}$ ) a reflexionar desde experiencias empíricas sobre la manera en que el juego transforma consciente e inconscientemente la identidad y la cultura docentes en el terreno de la enseñanza de la Historia. Un hecho que está estrechamente relacionado con el intenso y creciente fomento del aprendizaje interdisciplinar y por competencias que, apoyadas en los propios marcos legislativos, están llevando hoy a cabo las diferentes entidades educativas (incluyendo aquí cualquier tipo de fórmula integradora, práctica y/o transversal de conocimiento). El Departamento de Historia, tomado por Fenwick, Minty y Priestley (2013) como ámbito de confluencia entre las políticas educativas y las prácticas innovadoras que se desean implementar, constituye a este respecto un elemento de análisis imprescindible.

Sin embargo, si ha habido una cuestión relacionada con la innovación docente que ha acaparado un mayor protagonismo en los últimos tiempos esa ha sido sin duda alguna la del TPACK (Technological Pedagogical Content Knowledge), acrónimo que sintetiza el tipo de conocimiento pedagógico característico de nuestra cultura tecnológica. En relación a este tema, han sido muchos los autores que en los últimos diez años han reivindicado la importancia de la formación (Doering, Veletsianos, Scharber y Miller, 2009; Wilson y Wright, 2010), la reflexión e incluso la mentorización entre profesores de Historia. Participando de una línea de investigación continuada en años posteriores (Callahan, Saye y Brush, 2015, 2016), así lo hacían por ejemplo Saye, Kohlmeier, Brush, Mitchell y Farmer (2009), quienes reivindicaban tales estrategias con el objetivo de establecer "una base de conocimientos profesionales de práctica inteligente", en torno a un estudio de caso mediante el cual analizaban las experiencias de seis docentes. Por su parte, Harris y Hofer (2011) centraban la cuestión del TPACK en la correcta planificación del trabajo, llegando a identificar el desarrollo de tres importantes mejoras gracias a ello: una mayor variedad y conciencia sobre los recursos utilizados por los docentes, un mayor compromiso intelectual de los estudiantes y, finalmente, una integración razonada y efectiva de las tecnologías educativas.

En su descripción de la clase de Historia de una docente, Van Vaerenewyck, Shinas y Steckel (2017) partían directamente de la base de que la perspectiva del TPACK como marco teórico era insuficiente para comprender las implicaciones que traía consigo dicha integración, por lo que preferían optar por el "TPACK+", una suerte de versión expandida de dicho concepto que pretendía albergar las circunstancias socioculturales específicas del entorno educativo. Aunque escasas, autores como Sheffield (2011) o Swan y Hofer (2011) han planteado miradas algo más críticas sobre la implementación del TPACK. Estos últimos analizaban el uso del podcasting por parte de ocho profesores, llegando a la conclusión de que, si bien estos mostraron un gran conocimiento pedagógico (TPK), lo cierto es que carecían de un conocimiento previo del contenido tecnológico en sí mismo (TCK) a la hora de implementar este tipo de proyectos. Un hecho que, según autores como Semerci y Aydin (2018), iría estrechamente ligado a una actitud negativa producida por la falta de formación en esta línea, más que por cuestiones de género, edad o experiencia docente, del mismo modo que Bas y Senturk (2018) constataban desde su estudio cuantitativo la necesidad 
de aumentar los esfuerzos para conseguir una integración más efectiva de las NTICs por parte de los docentes turcos. En concreto, según el más que interesante trabajo de Riel, Lawless y Brown (2016), podríamos hablar hasta de seis grandes retos con los que los docentes se encuentran a la hora de enfrentar cualquier tipo de planteamiento innovador, dentro de los cuales se encontrarían a su vez varias categorías distintas: 1) las actividades del currículo y su correcta realización; 2) la implicación del estudiante; 3) los objetivos y expectativas curriculares; 4) la "orquestación curricular" o programación; 5) los retos fuera de la clase; y 6) los problemas tecnológicos.

Finalmente, merecería la pena destacar investigaciones como las de Milman y Bondie (2012) o la de Callahan, Saye y Brush (2015), quienes exploraban los materiales curriculares educativos digitales, preguntándose hasta qué punto el uso de estos podría ayudar a los profesores de Historia a desarrollar el tipo de conocimiento pedagógico que nuestra cultura tecnológica actual requeriría. Si bien los resultados obtenidos en el caso de los últimos autores señalados determinan que ninguno de los seis profesores investigados llegó a adoptar completamente una pedagogía novedosa, parece ser que el proceso investigador contribuyó al acercamiento entre profesores y estrategias tan interesantes como la investigación histórica basada en problemas. Desde la perspectiva de los autores, tal vinculación podría ser reforzada en el futuro mediante el fomento de experiencias dinámicas, de un currículo digital enfocado hacia la colaboración entre los distintos agentes educativos y la incorporación de mentores expertos que facilitasen los procesos de aprendizaje.

Así pues, podría concluirse que las transformaciones en la cultura docente derivadas de la implementación de nuevas estrategias didácticas en Historia requerirían, al igual que en cualquier otro ámbito educativo, una transformación paralela correspondiente con la adopción de una actitud proactiva y a la vez autocrítica respecto a los procesos educativos diseñados.

\subsection{Evaluación de la innovación}

En un cuarto y último grupo de trabajos nos encontramos todas aquellas investigaciones centradas en la evaluación global de la implementación de proyectos innovadores concretos en la clase de Historia, los cuales son normalmente abordados desde el punto de vista de su repercusión en el aprendizaje del alumnado. Al reunir un posicionamiento teórico, un despliegue metodológico (etnográfico y con estudio de casos en muchas ocasiones) y, lo más interesante, unas conclusiones extraídas directamente de la práctica, este tipo de estudios resultan clave tanto para terminar de conocer el trasfondo científico en el que se inscribe la temática elegida, como para establecer los límites y aspiraciones a la hora de plantear una nueva investigación. En la mayoría de ellos aparecen tres aspectos clave: un determinado programa de innovación, el contexto en que se implementa y el docente que establece los ajustes necesarios para ello. Así se ve claramente en trabajos como los de, por ejemplo: Jocson (2012), quien analizaba las repercusiones del proyecto multimedia Coming to California en un centro con gran presencia de estudiantes de diferentes etnias y orígenes humildes; Milner (2014), centrado en la actividad docente de la Sra. Shaw, experimentada profesora de Historia, con el objetivo de conocer su particular modo de incentivar la motivación del alumnado en un contexto de diversidad cultural; o Jaffee (2016), quien abordaba la enseñanza de la Historia para estudiantes latinos recién llegados al centro, por parte de un profesor que empleaba diferentes técnicas de investigación para diseñar sus programaciones.

En realidad, este último eje temático podría considerarse el más antiguo de los cuatro identificados, al tiempo que resulta el más transversal de todos, por lo que podría ser perfectamente integrado en cualquiera de los otros. Hace casi veinte años, investigaciones como la de Mingorance, Estebaranz y Marcelo (2000) analizaban ya el impacto de la innovación en general atendiendo a dimensiones como la organizativa, la didáctica y la curricular, mientras otras como la de García Quintanilla (2000), se centraban en la formación docente para la innovación desde la perspectiva, esta vez, de un centro de actualización y capacitación magisterial. Algo posterior sería el estudio de caso llevado a cabo por López Ruiz (2006) en un centro educativo de Sevilla, con el fin de rastrear las transformaciones derivadas de la implementación de dos programas de innovación para 
atender a la diversidad. En un plano metodológico, Harniss (2006) proponía en estas mismas fechas algunas orientaciones para la evaluación de lo que denominaba la "instrucción útil" en Historia, entendiendo por ello un tipo de enseñanza donde objetivos, instrucción y evaluación estuviesen perfectamente alineados. Más recientemente, la completísima obra editada por López Torres, García Ruiz y Sánchez Agustí (2018), una especie de antología a través de los casi treinta años del área de Didáctica de las Ciencias Sociales, alberga varios textos que ayudan a comprender la evolución teórica y metodológica de la investigación en torno a las consecuencias de la innovación, entre otros muchos temas.

Mención aparte merecerían una serie de trabajos que en los últimos tiempos han venido reflexionando sobre la manera de evaluar la eficacia de determinados aspectos del modelo educativo innovador desde la perspectiva del denominado "pensamiento histórico" (Gómez y Miralles, 2015; Gómez, Ortuño y Molina, 2014; Pagès, 2009). Como demuestran las numerosas investigaciones de una serie de autores españoles, podría decirse que el objetivo común ha sido concretar dicha perspectiva didáctica en competencias o destrezas evaluables (Domínguez, 2015; Gómez y Miralles, 2016; Miralles, Gómez y Rodríguez, 2017), acordes con el nuevo paradigma del aprendizaje competencial. Sáiz, por ejemplo, entendía de hecho el pensamiento histórico como "alfabetización histórica", contemplando "el planteamiento y solución de problemas, el análisis de fuentes históricas y la construcción de narrativas" (2013, p. 43), frente a las limitaciones del modelo educativo tradicional y los recursos a él asociados (Colomer, Sáiz y Valls, 2018; Sáiz, 2014). Con el fin de animar estas nuevas dimensiones, Molina y Ortuño (2018) subrayaban la importancia de la "empatía histórica", mientras que Miguel-Revilla y Sánchez Agustí (2018) se apoyaban en Duquette (2015) y la relación que establecía la autora con la idea de "conciencia histórica", para defender esta como categoría privilegiada para analizar las concepciones del alumnado sobre determinados contenidos de la materia de Historia. En esta línea, Bautista (2018) destacaba la necesidad de diseñar experiencias que favorecieran el desarrollo del pensamiento histórico como herramienta para la "contextualización curricular". En el plano internacional, autores como Greene, Bolick y Robertson (2010) han investigado las repercusiones del "hypermedia learning environment" (HLE) en el pensamiento histórico, reflexionando a su vez sobre las posibilidades de desarrollo en paralelo de lo que denominaban el "conocimiento declarativo", es decir, memorístico o positivo. Igual de interesante resulta el estudio de Meuwissen (2017) sobre el impacto del programa Teachers Doing History $(T D H)$ para impulsar la investigación histórica entre el alumnado, mediante una serie de actividades diseñadas en torno a la pregunta "¿cómo brindamos a los estudiantes nuevas oportunidades para desarrollar un pensamiento y entendimiento histórico poderoso a la vez que aliviamos los efectos de las condiciones que limitan nuestros esfuerzos?" (2017, p. 261). Junto a todo ello, han sido varios los autores que se han centrado en las transformaciones de la cultura docente, previas y simultáneas al trabajo con el pensamiento histórico (Ledman, 2015; Margrét, van Boxtel y van Drie, 2018)

Tanto en estos últimos trabajos como en los anteriormente citados se percibe que el elemento más común a toda la investigación en innovación didáctica es sin duda el optimismo mostrado por los autores respecto a los resultados obtenidos. Más allá de la afirmación de una mejora cualitativa de la enseñanza de la Historia, el aumento de la motivación y el compromiso de los discentes (Alongi, Heddy y Sinatra, 2016; Maguth, Yamaguchi y Elliott, 2010), así como de la adquisición de valores (Mathews, 2010), se suele presentar como la consecuencia lógica de la implementación de cualquier tipo de estrategia innovadora. Comparando el aprendizaje de la Historia con un libro de texto tradicional y una nueva modalidad basada en el trabajo con los "organizadores cognitivos" desarrollados con el software Inspiration 6, Boon, Burke, Fore y Spencer (2006) irían un paso más allá al plantear incluso la superación de los estudiantes del formato tradicional por parte del alumnado participante en un proceso innovador de carácter experimental. Sin embargo, sería preciso concluir este cuarto eje temático advirtiendo que, junto a la actitud pro-innovación que encarnan todos los estudios citados hasta el momento, existe también un grupo de trabajos que, aunque ciertamente reducido, se atreven a identificar determinados aspectos problemáticos de la innovación didáctica. 
Mathews (2016), por ejemplo, llegaba a la conclusión de que, si bien el proyecto "Investigación Participativa Digital" (DPR) garantizó que el alumnado participase activa y cooperativamente en oportunidades para el desarrollo de una conciencia global gracias al fotoperiodismo, lo cierto es que no aprendieron demasiados contenidos curriculares. De forma parecida, Friedman y Heafner (2007) descubrían y analizaban el hecho de que la implementación exitosa de un proyecto basado en la participación en el desarrollo de sitios web no llegara a traducirse en un éxito de aprendizaje medible con una prueba de conocimientos al uso.

En línea con lo ya comentado en los apartados anteriores, este tipo de investigaciones demuestra que la aplicación de cualquier tipo de estrategia innovadora en la enseñanza y el aprendizaje de la Historia debe complementarse con un análisis paralelo de la efectividad que trae consigo en la práctica, y que este debe estar abierto a la posibilidad de que el éxito sea parcial o directamente nulo. Así pues, toda investigación que tome como caso de estudio la innovación en la clase, tanto de Historia como de cualquier otra materia, debiera centrarse, no solo en la evolución del compromiso del alumno, sino también en el progreso académico de este y en el grado de autocrítica existente en la cultura docente del profesor implicado en el proceso.

\section{Reflexiones finales}

Lo primero que se deduce de la revisión bibliográfica en torno a la innovación en Historia aquí realizada es que dicha cuestión ha sido abordada desde puntos de vista muy dispares entre sí, si bien estrechamente interconectados. Como se ha sugerido, los cuatro ejes temáticos identificados a partir de la articulación de la propia búsqueda bibliográfica, y analizados atendiendo tanto a conceptos fundamentales como a experiencias que rastrean las implicaciones prácticas de los mismos, podrían concebirse como las principales dimensiones del modelo educativo predominante en la actualidad, no solo en lo relativo a la materia de Historia. En este sentido, todo aquel investigador o docente interesado en abordar las implicaciones prácticas del discurso innovador en cualquier plano o nivel académico, con independencia de que su objeto de estudio sea o no el discurso innovador propiamente dicho, deberá tener en cuenta las herramientas y metodologías empleadas en la experiencia que analice, el papel desempeñado en ella por el docente implicado, así como su evaluación global. Tales dimensiones no son ni mucho menos los únicos elementos existentes en los procesos de enseñanza y aprendizaje escolares, pero sí aquellos que a día de hoy están encauzando la manera en que es concebida y practicada la innovación. Por ello, es preciso confirmar que el proceder emergente adoptado a la hora de analizar el vasto corpus bibliográfico existente ha cumplido su objetivo de ofrecer unos primeros asideros teóricos para empezar a trabajar las preguntas de investigación planteadas en el proyecto global al que se adscribe esta aportación.

En segundo lugar, la observación de las relaciones entre ciertos conceptos y sus aplicaciones prácticas a través de los trabajos consultados permite catalogar la necesidad de seducir al alumnado como uno de los aspectos más controvertidos de la problemática abordada. Este está claramente conectado con el intenso y creciente fomento de un determinado tipo de aprendizaje tanto por parte de las instituciones educativas como, consciente o inconscientemente, por los propios investigadores. La enseñanza seductora por definición es aquella que tiene como objetivo garantizar un aprendizaje activo, competencial, experiencial, reflexivo, procesual, participativo, interactivo, creativo, cooperativo e interdisciplinar. A este fin se dirige precisamente el uso de las herramientas más acordes con los códigos audiovisuales actuales (Kadakia, 2005; Kili, 2005; Schul, 2012), con el ejemplo paradigmático de los videojuegos al frente, pero también, de forma complementaria, las metodologías que favorecen el trabajo con cuestiones sociales del presente, el trato personalizado, el desarrollo de la capacidad investigadora y del pensamiento histórico. Este trabajo reconoce expresamente el potencial de este último elemento, no solo como estrategia de seducción, sino también como criterio de evaluación del éxito o fracaso de una medida innovadora en la clase de Historia. En la línea de lo planteado por algunos de los investigadores citados, y dentro del gran reto educativo que supone el diseño de instrumentos y métodos de evaluación propios del siglo XXI, 
resulta urgente comenzar a reformular la didáctica de la Historia en clave competencial.

A raíz de todo ello, debemos afirmar, en tercer lugar, que los docentes más desafiantes y que atraen más la atención del alumnado, aquellos más afines al modelo innovador, son los grandes protagonistas de los estudios científicos. Debido además a la madurez que se les presupone respecto a los discentes, son de hecho aquellos cuyas opiniones poseen un mayor interés para los investigadores, siendo a menudo privilegiadas por encima de cualquier otra. Así, por ejemplo, son muy pocas las investigaciones que tienen en consideración la perspectiva del alumnado; un hecho especialmente alarmante a la hora de trabajar temas como, por ejemplo, la aplicación de los videojuegos en la clase de Historia (Egea, Arias y García, 2017; Martínez, Egea y Arias, 2018). En última instancia, esto invita a sumar la perspectiva del alumnado al resto de dimensiones temáticas aquí identificadas, completando así un enfoque teórico complejizador de los procesos innovadores. Después de todo, el docente no es ni mucho menos el único responsable del éxito de la innovación, sino tan solo un agente más cuyo nivel de motivación es tan importante como el del alumnado. Por ello resulta imprescindible comenzar a observar la medida en que el profesor de Historia aspira verdaderamente a disolver la manera superficial de emplear los medios, inculcando en sus estudiantes un proceso de metacognición que trascienda el conocimiento instrumental para aspirar a una determinada sensibilización ética. Hasta ahora, aspectos como este último han permanecido ajenos a la mirada de los investigadores como consecuencia de una excesiva focalización en cuestiones elementales y en efectos cortoplacistas de la innovación que hace que las perspectivas críticas sobre la transformación de la cultura docente en relación a este fenómeno, o, en general, sobre los resultados de los procesos innovadores, sean insuficientes. Salvo honrosas excepciones (Friedman y Heafner, 2007; Mathews, 2016; Sheffield, 2011; Swan y Hofer, 2011), la mayoría de las investigaciones aquí aludidas priman los beneficios del uso educativo de las NTICs, omitiendo, desatendiendo o minusvalorando las posibles dificultades en general que pueden llegar a generar con el tiempo en la práctica diaria ${ }^{3}$.

Por todo ello, este trabajo defiende finalmente la urgencia de empezar a contrarrestar una forma tanto de enseñar Historia como de investigar su didáctica basada en la defensa acrítica del aprendizaje competencial, de la autonomía del discente y del uso de las NTICs, amparada hoy en las leyes educativas, en los diferentes medios y plataformas de divulgación, pero también en la propia producción científica. Frente a ello, resulta imprescindible plantear investigaciones empíricas que no se limiten a subrayar las virtudes de la innovación, sino que también constaten todas aquellas imprecisiones y utopías que emanan hoy de su choque con la realidad de la escuela. Así, por ejemplo, sería preciso cuestionarse si la autonomía que el discurso legislativo concede al docente significa realmente independencia y no más bien autogestión, es decir, sometimiento ante un aumento históricamente inédito de la carga de trabajo y de responsabilidades frente a los procesos innovadores. Si de verdad ha de existir un interés investigador por la figura del docente o por cualquier otro aspecto relacionado con la didáctica de la Historia en la actualidad, este debe interpretarse sobre el trasfondo de las múltiples contradicciones emergidas hoy a la hora de llevar a la práctica el discurso global de la innovación. Ni el exceso de optimismo de los investigadores ni el exceso de pesimismo de algunos profesores deben impedir el desarrollo de reflexiones críticas y autocríticas en este sentido. Un proceso ralentizado sin duda por el hecho de que la innovación en didáctica de la Historia no conforme aún ningún campo específico de investigación, constituyendo simplemente una cuestión transversal a otras tantas de carácter principal (currículo, epistemología, evaluación del aprendizaje, etc.). Por ello debemos reclamar hoy un lugar propio para la innovación en didáctica de la Historia que se sitúe entre la reflexividad y la investigación aplicada en temáticas

3 Las conclusiones de Wahyuningtyas y Ratnawati (2016) ejemplifican a la perfección esta consideración: "Based on research result, instructional activity using multimedia is very interesting, pleasing, and effective. Beside information and knowledge in the multimedia is up-to-date and contextual. It is not surprising if students' response in the instructional process is full of enthusiasm. When their teacher were explaining instructional material by multimedia help, students showed discipline, active to ask and respond" (p. 170). 
que sí han conseguido ya consolidarse: la evaluación, el patrimonio, la identidad, la ciudadanía o la formación y las concepciones del profesorado (Miralles y Rodríguez, 2015).

Deacuerdo precisamente a la existencia de unas relativamente escasas pero bien argumentadas voces críticas, el "dispositivo" educativo predominante, recurriendo a la metáfora reivindicada por Michel Foucault, no constituye ni mucho menos un escenario homogéneo y armonioso, sino, al contrario, un enfrentamiento tensional entre dos modelos discursivos y prácticos bien distintos: por un lado, la moda innovadora ha conseguido ya convencer a todo tipo de agentes educativos -incluyendo a la mayoría de los investigadores- e instalarse en la escuela y en la academia; por otro, no debemos obviar que el modelo tradicional (vinculado al enciclopedismo, a las clases magistrales, a la pasividad, a la memorística, a lo cuantitativo, al examen como mecanismo de estratificación social, etc.) sigue representando para muchas personas un marco referencial con el que comparar los resultados de la innovación, e incluso, en los casos más extremos, el único camino para alcanzar la calidad educativa. Esto hace que estemos en el momento más oportuno para formular otras muchas cuestiones que, en algunos casos, abrirán nuevos procesos de revisión bibliográfica: ¿de qué formas se manifiestan las tensiones discursivas existentes entre los dos modelos educativos hoy confluyentes en la práctica?, ¿cuáles son las consecuencias epistemológicas de la innovación actual en la didáctica de la Historia?, ¿qué relación guarda la innovación metodológica con las renovaciones historiográficas más recientes?, ¿qué conclusiones se extraerían del análisis de dos casos de estudio distintos a partir de una misma metodología inspirada en las dimensiones aquí identificadas?, ¿de qué manera es preciso actualizar o transformar el modelo TPACK a partir de los resultados de esta investigación?, ¿cómo es recibida la innovación por parte del alumnado y qué papel juega en este proceso la cultura escolar familiar?, ¿qué repercusiones socioculturales tiene la activación del alumnado por medio del desarrollo de las capacidades investigadoras a partir del fomento del pensamiento histórico?

Así pues, los resultados obtenidos tras este proceso de revisión bibliográfica tan solo representan un punto de partida, válido para otros investigadores y docentes de Historia interesados en emprender sus propios procesos de mejora y superar la disyuntiva tradición-innovación.

\section{Bibliografía}

Abdu-Raheem, O. B. (2012). Effects of Problem-Solving Method on Secondary School Students' Achievement and Retention in Social Studies, in Ekiti State, Nigeria. Journal of International Education Research, 8(1), 19-26. https://doi.org/10.19030/jier.v8i1.6692

Adeyemi, B. A. (2008). Effects of Cooperative Learning and Problem-Solving Strategies on Junior Secondary School Students' Achievement in Social Studies. Electronic Journal of Research in Educational Psychology, 6(3), 691-708.

Alongi, M. D., Heddy, B. C. y Sinatra, G. M. (2016). Real-World Engagement with Controversial Issues in History and Social Studies: Teaching for Transformative Experiences and Conceptual Change. Journal of Social Science Education, 15(2), 26-41. https://doi.org/10.4119/UNIBI/ jsse-v15-i2-1479

Anderman, L. H., Andrzejewski, C. E. y Allen, J. (2011). How Do Teachers Support Students' Motivation and Learning in Their Classrooms? Teachers College Record, 113(5), 969-1003.

Ansó, M. B. (2017). Pedagogías lúdicas de innovación. Buenas prácticas de enseñanza con juegos digitales [tesis doctoral], R. Yuste y G. Esnaola (Dirs.). Universidad de Extremadura. http://hdl. handle.net/10662/6319

Aponte-Martínez, G. J. y Pellegrino, A. (2017). Youthscaping Our Classrooms: Nurturing Youth Civic Agency through Youth-Centered Pedagogies. Social Studies, 108(3), 99-110. https://doi.org/1 $\underline{0.1080 / 00377996.2017 .1324391}$

Barrow, E., Anderson, J. y Honer, M. (2017). The Role of Photoblogs in Social Studies Classroom: Learning about the People of the Civil War. Contemporary Issues in Technology and Teacher Education, 17(4), 504-521. 
Bas, G. y Senturk, C. (2018). An Evaluation of Technological Pedagogical Content Knowledge (TPACK) of In-Service Teachers: A Study in Turkish Public Schools. International Journal of Educational Technology, 5(2) 46-58.

Bates, A. (2008). Learning to Design WebQuests: An Exploration in Preservice Social Studies Education. Journal of Social Studies Research, 32(1), 10-21.

Binkley, R., Keiser, M. y Strahan, D. (2011). Connected Coaching: How Three Middle School Teachers Responded to the Challenge to Integrate Social Studies and Literacy. Journal of Social Studies Research, 35(2), 131-162.

Boardman, A., Buckley, P., Vaughn, S., Roberts, G., Scornavacco, K. y Klingner, J. K. (2016). The Relationship between Implementation of Collaborative Strategic Reading and Student Outcomes for Adolescents with Disabilities. Journal of Learning Disabilities, 49(6), 644-657. https://doi.org/10.1177/0022219416640784

Bolick, Ch. M. (2006). Digital Archives: Democratizing the Doing of History. International Journal of Social Education, 21(1), 122-134.

Boon, R. T., Burke, M. D., Fore, C. y Spencer, V. G. (2006). The Impact of Cognitive Organizers and Technology-Based Practices on Student Success in Secondary Social Studies Classrooms. Journal of Special Education Technology, 21(1), 5-15. https://doi. org/10.1177/016264340602100101

Boon, R. T., Fore, C. y Spencer, V. G. (2007). Teachers' Attitudes and Perceptions toward the Use of Inspiration 6 Software in Inclusive World History Classes at the Secondary Level. Journal of Instructional Psychology, 34(3), 166-171.

Brewer, E. A. y Fritzer, P. (2011). Teaching Students to Infer Meaning through Material Culture. Clearing House: A Journal of Educational Strategies, Issues and Ideas, 84(2), 43-46. https:// doi.org/10.1080/00098655.2010.507824

Bull, P. H. y Adams, S. (2012). Learning Technologies: Tweeting in a High School Social Studies Class. Journal of Educational Technology, 8(4), 26-33. https://doi.org/10.26634/jet.8.4.1644

Bull, G., Hammond, T. y Ferster, B. (2008). Developing Web 2.0 Tools for Support of Historical Inquiry in Social Studies. Computers in the Schools, 25(3-4), 275-287. https://doi. org/10.1080/07380560802367761

Butchart, S., Forster, D., Gold, I., Bigelow, J., Korb, K., Oppy, G. y Serrenti, A. (2009). Improving Critical Thinking Using Web Based Argument Mapping Exercises With Automated Feedback. Australasian Journal of Educational Technology, 25(2), 268-291. https://doi.org/10.14742/ ajet. 1154

Callahan, C., Saye, J. y Brush, T. (2015). Supporting In-Service Teachers' Professional Teaching Knowledge with Educatively Scaffolded Digital Curriculum. Contemporary Issues in Technology and Teacher Education (CITE Journal), 15(4), 568-599.

Callahan, C., Saye, J. y Brush, T. (2016). Interactive and Collaborative Professional Development for In-Service History Teachers. Social Studies, 107(6), 227-243. https://doi.org/10.1080/0037 $\underline{7996.2016 .1214905}$

Castek, J., Coiro, J., Guzniczak, L. y Bradshaw, C. (2012). Examining Peer Collaboration in Online Inquiry. Educational Forum, 76(4), 479-496. https://doi.org/10.1080/00131725.2012.707756

Cengelci, T. (2013). Social Studies Teachers' Views on Learning outside the Classroom. Educational Sciences: Theory and Practice, 13(3), 1836-1841.

Chee, Y. S., Mehrotra, S. y Liu, Q. (2013). Effective Game Based Citizenship Education in the Age of New Media. Electronic Journal of e-Learning, 11(1), 16-28.

Chee, Y. S., Mehrotra, S. y Ong, J. C. (2015a). Authentic Game-Based Learning and Teachers' Dilemmas in Reconstructing Professional Practice. Learning, Media and Technology, 40(4), 514-535. https://doi.org/10.1080/17439884.2014.953958

Chee, Y. S., Mehrotra, S. y Ong, J. C. (2015b). Professional Development for Scaling Pedagogical Innovation in the Context of Game-Based Learning: Teacher Identity as Cornerstone in "Shifting" Practice. Asia-Pacific Journal of Teacher Education, 43(5), 423-437. https://doi.org/1 


\section{$\underline{0.1080 / 1359866 \times .2014 .962484}$}

Chen, Ch. J. (2006). The Design, Development and Evaluation of a Virtual Reality Based Learning Environment. Australasian Journal of Educational Technology, 22(1), 39-63. https://doi. org/10.14742/ajet.1306

Cheong, Ch. M. y Cheung, W. S. (2008). Online Discussion and Critical Thinking Skills: A Case Study in a Singapore Secondary School. Australasian Journal of Educational Technology, 24(5), 556-573. https://doi.org/10.14742/ajet.1191

Cheung, W. S. y Hew, K. F. (2009). A Review of Research Methodologies Used in Studies on Mobile Handheld Devices in K-12 and Higher Education Settings. Australasian Journal of Educational Technology, 25(2), 153-183. https://doi.org/10.14742/ajet.1148

Colomer, J. C., Sáiz, J., Valls, R. (2018). Competencias históricas y actividades con recursos tecnológicos en libros de texto de Historia: nuevos materiales y viejas rutinas. Ensayos: Revista de la Facultad de Educación de Albacete, 33(1), 53-64. http://dx.doi.org/10.18239/ ensayos.v33i1.1740

Connor, D. J. y Lagares, C. (2007). Facing High Stakes in High School: 25 Successful Strategies from an Inclusive Social Studies Classroom. TEACHING Exceptional Children, 40(2), 18-27. https://doi.org/10.1177/004005990704000203

Crocco, M. S. y Cramer, J. (2005). Women, Webquests, and Controversial Issues in the Social Studies. Social Education, 69(4), 143-148.

Cuenca, J. M. y Martín, M. J. (2010). Virtual Games in Social Science Education. Computers \& Education, 55(3), 1336-1345.

Daniels, M. L. (2010). A Living History Classroom Using Re-Enactment to Enhance Learning. Social Education, 74(3), 135-136.

De Frutos, J. I. (2015). Juegos de simulación en el aula: una práctica educativa que fomenta el pensamiento histórico [tesis doctoral], E. Gómez Rodríguez (Dir.). Málaga: Universidad de Málaga. http://hdl.handle.net/10630/12617

De Miguel, R. (2014). La innovación didáctica en la enseñanza-aprendizaje de la geografía en Educación Secundaria [tesis doctoral], M. Sánchez Agustí (Dir.). Universidad de Valladolid. http://uvadoc.uva.es/handle/10324/7418

Devlin-Scherer, R. y Sardone, N. B. (2010). Digital Simulation Games for Social Studies Classrooms. Clearing House: A Journal of Educational Strategies, Issues and Ideas, 83(4), 138-144. https:// doi.org/10.1080/00098651003774836

Diacopoulos, M. M. (2015). Untangling Web 2.0: Charting Web 2.0 Tools, the NCSS Guidelines for Effective Use of Technology, and Bloom's Taxonomy. Social Studies, 106(4), 139-148. https:// doi.org/10.1080/00377996.2015.1015711

Doering, A., Veletsianos, G., Scharber, C. y Miller, C. (2009). Using the Technological, Pedagogical, and Content Knowledge Framework to Design Online Learning Environments and Professional Development. Journal of Educational Computing Research, 41(3), 319-346. https://doi. org/10.2190/EC.41.3.d

Domínguez, J. (2015). Pensamiento histórico y evaluación de competencias. Barcelona: Graó.

Duarte, O. (2015). La enseñanza de la Historia en la Educación Secundaria: Innovación, cambio y continuidad [tesis doctoral], F. J. Merchán Iglesias (Dir.). Universidad de Sevilla. http://hdl. handle.net/11441/30778

Duquette, C. (2015). Relating Historical Consciousness to Historical Thinking Through Assessment. En K. Ercikan y P. Seixas (Eds.), New Directions in Assessing Historical Thinking (pp. 51-63). New York: Routledge.

Ediger, M. (2011). Learning Stations in the Social Studies. Education, 131(3), 467-470.

Egea, A., Arias, L. y García, A. J. (2017). Videojuegos, historia y patrimonio: primeros resultados de una investigación educativa evaluativa en educación secundaria. Revista Interuniversitaria de Investigación en Tecnología Educativa, 2, 28-40. https://doi.org/10.6018/riite/2017/283801

Egea, A., Arias, L. y J. Santacana (coords.) (2018). Y la arqueología llegó al aula: la cultura material 
y el método arqueológico para la enseñanza de la historia y el patrimonio. Gijón: Ediciones Trea. https://doi.org/10.17398/1695-288X.17.1.61

Elliott, K. y Sweeney, K. (2008). Quantifyng the Reuse of Leaning Objects. Australasian Journal of Educational Technology, 24(2), 137-142. https://doi.org/10.14742/ajet.1216

Ellison, K. y Matthews, C. (2010). Virtual History: A Socially Networked Pedagogy of Enlightenment. Educational Research, 52(3) 297-307. https://doi.org/10.1080/00131881.2010.504065

Fenwick, A. J., Minty, S. y Priestley, M. (2013). Swimming against the Tide: A Case Study of an Integrated Social Studies Department. Curriculum Journal, 24(3), 454-474. https://doi.org/10 $.1080 / 09585176.2013 .805658$

Fernández, M. D. (2016). La innovación educativa a través de buenas prácticas con TIC en los centros de Primaria y Secundaria del Campo de Gibraltar [tesis doctoral], J. De Pablo y F. Pavón (Dirs.). Universidad de Cádiz.

Foels, S. (2009). Big Expectations: Big Ideas in Honors and Inclusion Classes. Journal of Inquiry and Action in Education, 2(1), 74-90.

Friedman, A. M. y Heafner, T. L. (2007). "You Think for Me, so I Don't Have To". The Effect of a Technology-Enhanced, Inquiry Learning Environment on Student Learning in 11th-Grade United States History. Contemporary Issues in Technology and Teacher Education (CITE Journal), 7(3), 199-216.

Gallavan, N. P. y Kottler, E. (2007). Eight Types of Graphic Organizers for Empowering Social Studies Students and Teachers. Social Studies, 98(3), 117-123. https://doi.org/10.3200/ TSSS.98.3.117-128

García Quintanilla, M. (2000). Cambio y resistencias en el proceso de innovación educativa [tesis doctoral], J. Tejada (Dir.). Universidad Autónoma de Barcelona.

Garran, D. K. (2008). Implementing Project-Based Learning to Create "Authentic" Sources: The Egyptological Excavation and Imperial Scrapbook Projects at the Cape Cod Lighthouse Charter School. History Teacher, 41(3), 379-389.

Gkatzidou, S. y Pearson, E. (2009). The Potential for Adaptable Accessible Learning Objecs: A Case Study in Accessible Vodcasting. Australasian Journal of Educational Technology, 25(2), 292307.

Gómez, C. J. y Miralles, P. (2015). ¿Pensar históricamente o memorizar el pasado? La evaluación de los contenidos históricos en la educación obligatoria en España. Revista de Estudios Sociales, 52, 52-68. http://dx.doi.org/10.7440/res52.2015.04

Gómez, C. J. y Miralles, P. (2016). Las competencias históricas en educación obligatoria: evaluación, estrategias basadas en la indagación, y argumentación de los estudiantes. Journal of New Approaches in Educational Research, 5(2), 139-146. https://doi.org/10.7821/naer.2016.7.172

Gómez, C. J., Ortuño, J. y Molina, S. (2014). Aprender a pensar históricamente. Retos para la historia en el siglo XXI. Revista Tempo e Argumento, 6(11), 5-27. http://dx.doi. org/10.5965/2175180306112014005

Grant, G. S. y Gradwell, J. M. (2009). The Road to Ambitious Teaching: Creating Big Idea Units in History Classes. Journal of Inquiry and Action in Education, 2(1), 1-26.

Greene, J. A., Bolick, Ch. M. y Robertson, J. (2010). Fostering historical knowledge and thinking skills using hypermedia learning environments: The role of self-regulated learning. Computers \& Education, 54, 230-243. https://doi:10.1016/j.compedu.2009.08.006

Harniss, M. K. (2006). Instructionally Useful Assessment in the Social Studies. Assessment for Effective Intervention, 31(2), 23-37.

Haro, P. (2015). Evaluando la innovación educativa con TIC en centros educativos. Un estudio de caso [tesis doctoral], M. Cebrián (Dir.). Universidad de Málaga. http://hdl.handle.net/10630/10039

Harris, J. B. y Hofer, M. J. (2011). Technological Pedagogical Content Knowledge (TPACK) in Action: A Descriptive Study of Secondary Teachers' Curriculum-Based, Technology-Related Instructional Planning. Journal of Research on Technology in Education, 43(3), 211-229. https://doi.org/10.1080/15391523.2011.10782570 
Haughey, M. y Muirhead, B. (2005). The Pedagogical and Multimedia Designs of Learning Objects for Schools. Australasian Journal of Educational Technology, 21(4), 470-490.

Haydon, T., Maheady, L. y Hunter, W. (2010). Effects of Numbered Heads Together on the Daily Quiz Scores and On-Task Behavior of Students with Disabilities. Journal of Behavioral Education, 19(3), 222-238. https://doi.org/10.1007/s10864-010-9108-3

Heafner, T. L. y Friedman, A. M. (2008). Wikis and Constructivism in Secondary Social Studies: Fostering a Deeper Understanding. Computers in the Schools, 25(3-4), 288-302.

Hernández-Ramos, P. y De la Paz, S. (2009). Learning History in Middle School by Designing Multimedia in a Project-Based Learning Experience. Journal of Research on Technology in Education, 42(2), 151-173.

Jaffee, A. T. (2016). Community, Voice, and Inquiry: Teaching Global History for English Language Learners. Social Studies, 107(3), 1-13. https://doi.org/10.1080/00377996.2016.1140626

Jocson, K. M. (2012). Youth Media as Narrative Assemblage: Examining New Literacies at an Urban High School. Pedagogies: An International Journal, 7(4), 298-316. https://doi.org/10.1080/155 4480X.2012.715735

Kadakia, M. (2005). Increasing Student Engagement by Using Morrowind to Analyze Choices and Consequences. TechTrends: Linking Research \& Practice to Improve Learning, 49(5), 29-32. https://doi.org/10.1007/BF02763687

Kearney, M. y Schuck, S. (2006). Spotlight on Authentic Learning: Student Developed Digital Video Projects. Australasian Journal of Educational Technology, 22(2), 189-208. https://doi. org/10.14742/ajet.1298

Keeler, C. G. (2008). When Curriculum and Technology Meet: Technology Integration in Methods Courses. Journal of Computing in Teacher Education, 25(1), 23-30.

Kiili, K. (2005). Participatory Multimedia Learning: Engaging Learners. Australasian Journal of Educational Technology, 21(3), 303-322. https://doi.org/10.14742/ajet.1322

King-Yin, S. y Trinidad, S. (2005). Using ICQ to Enhance Teacher-Student Relationships. Australasian Journal of Educational Technology, 21(4), 427-445. https://doi.org/10.14742/ajet.1313

Kingsley, K. V. y Boone, R. (2008). Effects of Multimedia Software on Achievement of Middle School Students in an American History Class. Journal of Research on Technology in Education, 41(2), 203-221. https://doi.org/10.1080/15391523.2008.10782529

Kingsley, K. V. y Brinkerhoff, J. (2011). Web 2.0 Tools for Authentic Instruction, Learning, and Assessment. Social Studies and the Young Learner, 23(3), 9-13.

Kracl, C. L. (2012). Review or True? Using Higher-Level Thinking Questions in Social Studies Instruction. Social Studies, 103(2), 57-60. https://doi.org/10.1080/00377996.2011.586382

Ledman, K. (2015). Navigating historical thinking in a vocational setting: teachers interpreting a history curriculum for students in vocational secondary education. Journal of Curriculum Studies, 47(1), 77-93. https://doi.org/10.1080/00220272.2014.984766

Lee, J. K. y Probert, J. (2010). Civilization III and Whole-Class Play in High School Social Studies. Journal of Social Studies Research, 34(1), 1-28.

Lim, K. Y. y Wang, J. Y. (2005). Collaborative Handheld Gaming in Education. Educational Media International, 42(4), 351-359. https://doi.org/10.1080/09523980500237765

Lipscomb, G. B., Guenther, L. M. y McLeod, P. (2007). Sounds Good to Me: Using Digital Audio in the Social Studies Classroom. Social Education, 71(3), 120-124.

López Ruiz, J. I. (2006). ¿Cómo evoluciona una innovación?: un estudio de caso. Kikiriki. Cooperación educativa, 81, 71-74.

López Torres, E., García Ruíz, C. R. y Sánchez Agustí, M. (2018). Buscando formas de enseñar: investigar para innovar en Didáctica de las Ciencias Sociales. Valladolid: Asociación Universitaria del Profesorado de Didáctica de las Ciencias Sociales, Universidad de Valladolid.

Maguth, B. M., Yamaguchi, M. y Elliott, J. (2010). Researching, Producing, Presenting: Students' Use of Technology for Global Advocacy in the Social Studies. Social Education, 74(2), 105106. 
Maquilón, J. J., García-Sanz, M. P. y Belmonte, M. L. (coords.). (2015). Innovación educativa en la enseñanza formal. Murcia: Universidad de Murcia, Asociación Universitaria de Formación del Profesorado.

Marcelo, C. (Dir.). (2011). Estudio de campo sobre la innovación educativa en los centros escolares. En IFIIE, Estudio sobre la innovación educativa en España. Innovación $N^{\circ}$ 17. Gobierno de España, Ministerio de Educación: Instituto de Formación del Profesorado, Investigación e Innovación Educativa (IFIIE), Centro Nacional de Innovación e Investigación Educativa (CNIIE).

Margrét, S., van Boxtel, C. y van Drie, J. (2018). Teaching historical thinking and reasoning: construction of an observation instrument. British Educational Research Journal, 44(6), 960981. https://doi.org/10.1002/berj.3471

Martell, C. C. y Sequenzia, M. R. (2016). Working the Dialectic: Teaching and Learning Teacher Research in Social Studies. Educational Forum, 80(4), 407-416. https://doi.org/10.1080/0013 1725.2016.1206156

Martínez, J. M., Egea, A. y Arias, L. (2018). Evaluación de un videojuego educativo de contenido histórico. La opinión de los estudiantes. RELATEC: Revista Latinoamericana de Tecnología Educativa, 17(1), 62-75.

Mathews, J. M. (2010). Using a Studio-Based Pedagogy to Engage Students in the Design of MobileBased Media. English Teaching: Practice and Critique, 9(1), 87-102.

Mathews, S. A. (2016). Using Digital Participatory Research to Foster Glocal Competence: Constructing Multimedia Projects as a Form of Global and Civic Citizenship. Journal of Social Studies Education Research, 7(2), 1-29.

Mazur, A. D., Brown, B. y Jacobsen, M. (2015). Learning Designs Using Flipped Classroom Instruction (Conception d'apprentissage à l'aide de l'instruction en classe inversée). Canadian Journal of Learning and Technology, 41(2), 26. http://dx.doi.org/10.21432/T2PG7P

Medina, A. M. (Coord.) (2015). Innovación de la educación y de la docencia. Madrid: Centro de Estudios Ramón Areces.

Meuwissen, K. W. (2017). "Happy Professional Development at an Unhappy Time": Learning to Teach for Historical Thinking in a High-Pressure Accountability Context. Theory \& Research in Social Education, 45, 248-285. https://doi.org/10.1080/00933104.2016.1232208

Miguel-Revilla, D. y Sánchez Agustí, M. (2018). Modelos de conciencia histórica en el alumnado de Educación Secundaria: tradición, simbología y contextualización en torno a los restos del franquismo. Panta Rei. Revista Digital de Ciencia y Didáctica de la Historia, 119-142. https:// doi.org/10.6018/pantarei/2018/6

Milman, N. B. y Bondie, R. (2012). An Examination of Teachers' Ratings of Lesson Plans Using Digital Primary Sources. Contemporary Issues in Technology and Teacher Education (CITE Journal), 12(4), 391-407.

Milner, R. H. (2014). Culturally Relevant, Purpose-Driven Learning \& Teaching in a Middle School Social Studies Classroom. Multicultural Education, 21(2), 9-17.

Mingorance, P., Estebaranz, A. y Marcelo, C. (2000). El impacto de la innovación a través del estudio de casos. Bordón. Revista de pedagogía, 52(2), 179-188.

Miralles, P., Gómez, C. J., Rodríguez, R. A. (coords.) (2017). La enseñanza de la historia en el siglo XXI: desarrollo y evaluación de competencias históricas para una ciudadanía democrática. Murcia: Edit.um, Ediciones de la Universidad de Murcia.

Miralles, P. y Rodríguez, R. A. (2015). Estado de la cuestión sobre la investigación en didáctica de la Historia en España. Índice histórico español, 128, 67-90.

Molina, S. y Ortuño, J. (2018). Evaluar la adquisición de las competencias históricas en Secundaria: un estudio de caso centrado en la empatía histórica. ENSAYOS, Revista de la Facultad de Educación de Albacete, 33(1), 1-22. http://dx.doi.org/10.18239/ensayos.v33i1.1737

O'Brien, Ch. y Wood, Ch. L. (2011). Video Modeling of Cooperative Discussion Group Behaviors with Students with Learning Disabilities in a Secondary Content-area Classroom. Journal of 
Special Education Technology, 26(4), 25-40. https://doi.org/10.1177/016264341102600403

Olukayode, A. S. y Salako, E. T. (2014). Effect of Jigsaw Technique and Gender on Students' Attitude to Ethnic Integration and Sustainable Development in Nigeria. World Journal of Education, 4(3), 46-52. https://doi.org/10.5430/wje.v4n3p46

Pagès, J. (2009). El desarrollo del pensamiento histórico como requisito para la formación democrática de la ciudadanía. Reseñas de Enseñanza de la historia, 7, 69-91.

Pagnotti, J. y Russell, W. B. (2012). Using Civilization IV to Engage Students in World History Content. Social Studies, 103(1), 39-48. https://doi.org/10.1080/00377996.2011.558940

Parry, D. (2008). Twitter for Academia [online]. Disponible en: <http://www.outsidethetext.com/2008/ twitter-for-academia/> [Último acceso: 13/04/2019].

Pineda-Alfonso, J. A. y García Pérez, F. F. (2011). La construcción de un ámbito de investigación escolar sobre el conflicto, la violencia y la guerra. Íber. Didáctica de las Ciencias Sociales, 68, 82-91.

Rauch, U., Cohodas, M. y Wang, T. (2009). The Arts 3D VLE Metaverse as a Network of Imagination. Innovate: Journal of Online Education, 5(6).

Riel, J., Lawless, K. A. y Brown, S. W. (2016). Listening to the Teachers: Using Weekly Online Teacher Logs for ROPD to Identify Teachers' Persistent Challenges When Implementing a Blended Learning Curriculum. Journal of Online Learning Research, 2(2), 169-200.

Riera, C. (2010). Un estudio etnográfico en el campo de la innovación educativa: el programa experimental aulas taller [tesis doctoral], J. Marrero y L. Feliciano (Dirs.). Universidad de La Laguna. http://riull.ull.es/xmlui/handle/915/9767

Rowland, N. (2010). Becoming a "History Person" or, If Sally Says It's Possible, It Must Be. Learning Disabilities: A Multidisciplinary Journal, 16(2), 97.

Sáiz, J. (2013). Alfabetización histórica y competencias básicas en libros de texto de historia y en aprendizajes de estudiantes. Didáctica de las Ciencias Experimentales y Sociales, 27, 43-66. https://doi.org/10.7203/dces.27.2648

Sáiz, J. (2014). Fuentes históricas y libros de texto en secundaria: una oportunidad perdida para enseñar competencias de pensamiento histórico. ENSAYOS, Revista de la Facultad de Educación de Albacete, 29(1), 83-99. http://dx.doi.org/10.18239/ensayos.v29i1.503

Salako, E. C., Eze, I. R. y Adu, E. O. (2013). Effects of Cooperative Learning on Junior Secondary School Students' Knowledge and Attitudes to Multicultural Education Concepts in Social Studies. Education, 133(3), 303-309.

Saye, J. W., Kohlmeier, J., Brush, T., Mitchell, L. y Farmer, C. (2009). Using Mentoring to Develop Professional Teaching Knowledge for Problem-Based Historical Inquiry. Theory and Research in Social Education, 37(1), 6-41. https://doi.org/10.1080/00933104.2009.10473386

Schul, J. E. (2012). Compositional Encounters: Evolvement of Secondary Students' Narratives While Making Historical Desktop Documentaries. Journal of Social Studies Research, 36(3), 219-244.

Schul, J. E. (2014). Film Pedagogy in the History Classroom: Desktop Documentary-Making Skills for History Teachers and Students. Social Studies, 105(1), 15-22. https://doi.org/10.1080/003 77996.2013 .788470

Semerci, A. y Aydin, M. K. (2018). Examining High School Teachers' Attitudes towards ICT Use in Education. International Journal of Progressive Education, 14(2), 93-105.

Sheffield, C. (2011). Navigating Access and Maintaining Established Practice: Social Studies Teachers' Technology Integration at Three Florida Middle Schools. Contemporary Issues in Technology and Teacher Education (CITE Journal), 11(3), 282-312.

Sheffield, C. y Swan, S. (2012). Digital Reenactments: Using Green Screen Technology to Recreate the Past. Social Education, 76(2), 92-95.

Snyder, C., Paska, L. M. y Besozzi, D. (2014). Cast from the Past: Using Screencasting in the Social Studies Classroom. Social Studies, 105(6), 310-314. https://doi.org/10.1080/00377996.2014. $\underline{951472}$ 
Stoddard, J. D. (2012). Film as a "Thoughtful" Medium for Teaching History. Learning, Media and Technology, 37(3), 271-288. https://doi.org/10.1080/17439884.2011.572976

Stoddard, J. D. y Marcus, A. S. (2010). More than "Showing What Happened": Exploring the Potential of Teaching History with Film. High School Journal, 93(2), 83-90.

Swan, K. y Hofer, M. (2011). In Search of Technological Pedagogical Content Knowledge: Teachers' Initial Foray into Podcasting in Economics. Journal of Research on Technology in Education, 44(1), 75-98. https://doi.org/10.1080/15391523.2011.10782580

Swan, K. y Hofer, M. (2013). Examining Student-Created Documentaries as a Mechanism for Engaging Students in Authentic Intellectual Work. Theory and Research in Social Education, 41(1), 133-175.

Swan, K., Hofer, M. y Swan, G. (2011). Examining authentic intelectual work with a social studies digital documentary inquiry project in a mandated state-testing environment. Journal of Digital Learning in Teacher Education, 27, 115-122. https://doi.org/10.1080/21532974.2011.1078466 $\underline{6}$

Swanson, E. y Wanzek, J. (2014). Applying Research in Reading Comprehension to Social Studies Instruction for Middle and High School Students. Intervention in School and Clinic, 49(3), 142147. https://doi.org/10.1177/1053451213496157

Tinnerman, L., Johnson, J. y Grimes, R. (2010). Technology Assisted Collaborative and ProjectBased Learning; of Blogs, Wikis, and Networking. Journal on School Educational Technology, 6(1), 1-6.

Van Vaerenewyck, L. M., Shinas, V. H. y Steckel, B. (2017). Sarah's Story: One Teacher's Enactment of TPACK+ in a History Classroom. Literacy Research and Instruction, 56(2), 158-175. https:// doi.org/10.1080/19388071.2016.1269267

Verezub, E., Grossi, V., Howard, K. y Watkins, P. (2008). Building E-Based Literacy for Vocational Education and Training Students. Australasian Journal of Educational Technology, 24(3), 326338. https://doi.org/10.14742/ajet.1212

Virtue, D. C., Buchanan y A., Vogler, K. E. (2012). Digging Postholes Adds Depth and Authenticity to a Shallow Curriculum. Social Studies, 103(6), 247-251. https://doi.org/10.1080/00377996.2 011.630699

Wahyuningtyas, N. y Ratnawati, N. (2016). Interactive Multimedia as Autonomous Learning Resource in the South Slope of Kelud Mountain in Blitar Regency. Journal of Education and Practice, 7(29), 168-171.

Wilson, E. y Wright, V. (2010). Images over Time: The Intersection of Social Studies through Technology, Content, and Pedagogy. Contemporary Issues in Technology and Teacher Education (CITE Journal), 10(2), 220-233.

Wilson, E. K., Wright, V., Inman, C. T. y Matherson, L. H. (2011). Retooling the Social Studies Classroom for the Current Generation. Social Studies, 102(2), 65-72.

Woelders, A. (2007). Using Film to Conduct Historical Inquiry with Middle School Students. History Teacher, 40(3), 363-396.

Worthington, T. A. (2018). Letting Students Control Their Own Learning: Using Games, Role-Plays, and Simulations in Middle School U.S. History Classrooms. Social Studies, 109(2), 136-150.

Wright-Maley, C. (2014). In Defense of Simulating Complex and Tragic Historical Episodes: A Measured Response to the Outcry over a New England Slavery Simulation. Canadian Social Studies, 47(1), 18-25.

Yukhymenko, M. A., Brown, S. W., Lawless, K. A., Brodowinska, K. y Mullin, G. (2014). Thematic Analysis of Teacher Instructional Practices and Student Responses in Middle School Classrooms with Problem-Based Learning Environment. Global Education Review, 1(3), 93110.

Yusmanto, H., Soetjipto, B. E. y Djatmika, E. T. (2017). The Application of Carousel Feedback and Round Table Cooperative Learning Models to Improve Student's Higher Order Thinking Skills (HOTS) and Social Studies Learning Outcomes. International Education Studies, 10(10), 39- 
49. https://doi.org/10.5539/ies.v10n10p39

Zhao, Y. (2007). Social Studies Teachers' Perspectives of Technology Integration. Journal of Technology and Teacher Education, 15(3), 311-333.

Zieger, L. y Farber, M. (2012). Civic Participation among Seventh-Grade Social Studies Students in Multi-User Virtual Environments. Journal of Interactive Learning Research, 23(4), 393-410. 



\section{Panta Rei}

PANTA REI es una revista digital de investigación orientada a la Historia y otras ciencias afines. Su principal objetivo es la transmisión del conocimiento científico, dando una oportunidad también a los jóvenes investigadores que quieren abrirse camino en el estudio de las ciencias humanas y sociales. Se compone de estudios originales relacionados con la disciplina histórica así como su didáctica y difusión. Las diferentes secciones que componen la revista son: artículos de investigación, entrevistas a profesionales, recensiones de monografías de actualidad y crónicas de congresos o eventos científicos relevantes.

Todos los artículos publicados son objeto de un proceso de revisión a cargo de un mínimo de dos evaluadores, que se consideran expertos en el ámbito temático del artículo propuesto. Nuestro deseo es poder ofrecer unos contenidos rigurosos, de calidad y de interés.

EI CEPOAT (Centro de Estudios del Próximo Oriente y la Antigüedad Tardía de la Universidad de Murcia) es la institución encargada de la coordinación y gestión de la revista, desde donde anualmente se lanzará la convocatoria para aquellos que estén interesados en publicar sus trabajos, siempre relacionados con la Historia, Arqueología, Historia del Arte, Didáctica de la Historia, etc.

PANTA REI is a digital journal focused on History and other sciences related to it. Its main objective is the transmission of scientific knowledge by giving also an opportunity to young researchers who want to make their way in the study of human and social sciences. It is composed by original studies related to History, as well as its didactics and promotion. The different sections of this journal are: research articles, interviews to professionals, recensions on monographs about current issues and reports about congresses or relevant scientific events.

All the articles published are subject to a revision process carried out by a minimum of two reviewers who are considered to be experts in the field of the article proposed. Our wish is to offer rigorous contents with quality and being of interest to the reader.

CEPOAT (Centre of Studies of the Middle East and Late Antiquity of the University of Murcia) is the institution in charge of the coordination and management of this journal. This is the centre from where the call for papers will be launched for all the people interested in publishing their papers, always related to History, Archeology, Art History, Teaching History, etc. 


\section{Normas de Publicación}

El autor se compromete a enviar trabajos originales, que no se encuentren publicados en otras revistas ni en otros idiomas. Así mismo, el mismo artículo no podrá ser presentado en otras revistas mientras dure el proceso de evaluación.

\section{Envío y presentación de originales}

Los artículos se enviarán exclusivamente a través del correo electrónico a la dirección pantarei@um.es. Los textos serán enviados en formato DOC y las imágenes en formato JPEG o TIFF, y con un tamaño mínimo de 2000 px. Éstas no aparecerán incorporadas en el texto, sino enviadas en archivo aparte y correctamente numeradas según su posición en el texto. Junto al trabajo, se rellenará y enviará un documento aparte en el que se especifiquen los datos del autor siguiendo el modelo disponible en la página Web de la revista.

Para la redacción de los trabajos se tendrá en cuenta el Manual de la American Psychological Association, en su sexta edición. La extensión máxima de los trabajos será de 30 páginas. La tipografía será Arial 11, con interlineado sencillo y sin espacio alguno entre párrafos. El texto deberá ir justificado a ambos márgenes y sin sangría en los primeros párrafos. Los márgenes serán de $2,50 \mathrm{~cm}$. En los casos en los que fuera necesario incorporar notas, éstas irán a pie de página, enumeradas consecutivamente, con tipografía Arial 10, interlineado sencillo y justificadas a ambos márgenes.

Una información más detallada se encuentra disponible en la página http://www.um.es/cepoat/ pantarei.

\section{Proceso de valoración y evaluación}

Una vez recibidos los trabajos, la Revista realizará una primera valoración. Si el trabajo enviado se ajusta a las normas de presentación propuestas, la temática es coincidente con la línea editorial de la revista y posee la calidad científica necesaria, será remitido al consejo asesor para una primera evaluación. Si no es así en este primer paso se puede rechazar directamente los documentos que incumplan claramente la línea editorial.

Será el Consejo Asesor quien indique a la revista la originalidad, relevancia, estructura, redacción, aparato bibliográfico, etc. del trabajo enviado y, para ello, se designará a dos revisores expertos externos que evaluarán cada uno de los trabajos, que pueden formar parte (o no) de este Consejo Asesor. La selección de los revisores se ajustará a la temática y características metodológicas del trabajo. El nombre y filiación de los autores serán eliminados del trabajo para su revisión, así como los revisores actuarán de manera anónima y confidencial.

Los revisores deberán rellenar un informe de evaluación que centrará su atención en aspectos tales como características formales, originalidad y novedad de los trabajos, relevancia de las propuestas y los resultados, calidad metodológica y validez científica.

Una vez terminado el proceso se decidirá la aceptación o no de los mismos y su publicación en el número que sea pertinente, así como las modificaciones susceptibles de ser realizadas para su final publicación. Dicha notificación se enviará únicamente por correo electrónico, en un plazo máximo de seis meses. 


\section{Publishing rules}

The author is committed to submit original papers not having been published in other reviews or in other languages. In this way, it is not allowed for the same paper to be presented in other reviews during the evaluation process.

\section{Submission and presentation of originals}

The articles will be exclusively submitted by email to pantarei@um.es. The texts will be submitted in DOC format and the images in JPEG or TIFF format, and with a minimum size of 2000 px. Images will not be integrated in the text but sent in another file and properly numbered according to their position in the text. Attached to the paper, a document will be filled out and sent where the author's data will be specified following the model available on the website.

The sixth edition of the Manual of the American Psychological Association will be taken into account for the writing of the papers. The length of the papers must not exceed 30 pages. Typography will be Arial 11 , with simple line spacing and no space between paragraphs. The text must be justified on both margins without indentation in the first paragraphs. Margins size will be $2.50 \mathrm{~cm}$. Where it could be necessary the incorporation of notes, they will be at the bottom of the page, consecutively numbered with typography Arial 10, simple line spacing and justified on both margins.

More detailed information is available on the website: http://www.um.es/cepoat/pantarei.

\section{Examination and assessment process}

The Journal will submit the papers to a first examination once received. If the paper follows the presentation guidelines, the subject agrees with the editorial line of this journal, and possess the scientific quality required, it will be sent to the advisory council for a first assessment. If not, the documents which clearly fail to complete the editorial line may be rejected straightaway in this first step.

The Advisory Council will indicate the originality, relevance, structure, writing, bibliography, etc. of the text to the journal; for this purpose, two outside experts will be designated to review the papers; these experts can be (or not) part of this Advisory Council. The selection of the experts will adjust to the subject and methodological characteristics of the paper. Name and affiliation of the author will be eliminated from the text for its review, in this way experts will act anonymously and confidentially.

The experts will fill out an assessment report which will focus on aspects such as formal characteristics, originality and novelty of the papers, relevance and results of the proposal, methodological quality and scientific validity.

Once the process is finished, the acceptance or not of the papers and its publication in the corresponding edition will be decided, as well as the modifications that may be done for its final publication. This notification will be sent by email within 6 months maximum. 


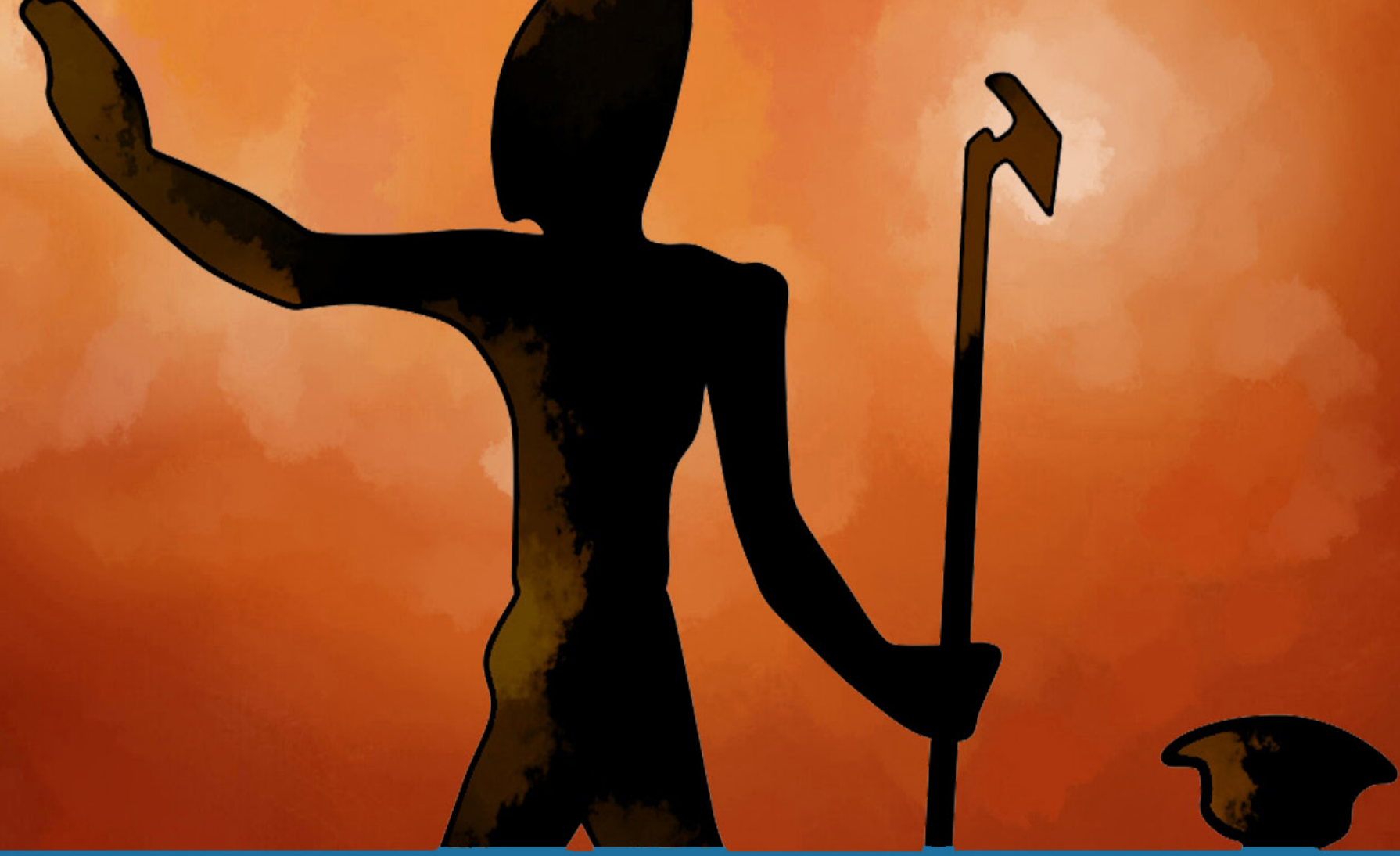

GepOA

centro de estudios de

próximo oriente y la

antigüedad tardía 